\title{
Using Artificial Neural Networks to Improve CFS Week 3-4 Precipitation and 2-Meter Air Temperature Forecasts
}

Yun Fan ${ }^{1}$, Vladimir Krasnopolsky ${ }^{2}$, Huug van den Dool ${ }^{1}$, Chung-Yu Wu ${ }^{1}$ and Jon Gottschalck ${ }^{1}$

Revised November, 2020

\author{
${ }^{1}$ Climate Prediction Center \\ ${ }^{2}$ Environmental Modeling Center \\ NOAA Center for Weather and Climate Prediction \\ 5830 University Research Court \\ College Park, Maryland 20740, USA \\ E-mail: Yun.Fan@noaa.gov
}

Early Online Release: This preliminary version has been accepted for publication in Weather and Forecasting, may be fully cited, and has been assigned DOI 10.1175/WAF-D-20-0014.1. The final typeset copyedited article will replace the EOR at the above DOI when it is published. 
2 Forecast skill from dynamical forecast models decreases quickly with projection time due to

3 various errors. Therefore, post-processing methods, from simple bias correction methods to more

4 complicated multiple linear regression-based Model Output Statistics, are used to improve raw

5 model forecasts. Usually, these methods show clear forecast improvement over the raw model

6 forecasts, especially for short-range weather forecasts. However, linear approaches have

7 limitations because the relationship between predictands and predictors may be nonlinear. This is

8 even truer for extended range forecasts, such as Week 3-4 forecasts.

9 In this study, neural network techniques are used to seek or model the relationships between a set 10 of predictors and predictands, and eventually to improve Week 3-4 precipitation and 2-meter 11 temperature forecasts made by the NOAA NCEP Climate Forecast System. Benefitting from

12 advances in machine learning techniques in recent years, more flexible and capable machine 13 learning algorithms and availability of big datasets enable us not only to explore nonlinear features 14 or relationships within a given large dataset, but also to extract more sophisticated pattern 15 relationships and co-variabilities hidden within the multi-dimensional predictors and predictands. 16 Then these more sophisticated relationships and high-level statistical information are used to 17 correct the model Week 3-4 precipitation and 2-meter temperature forecasts. The results show that 18 to some extent neural network techniques can significantly improve the Week 3-4 forecast accuracy and greatly increase the efficiency over the traditional multiple linear regression methods. 


\section{Introduction}

The public demand for sub-seasonal to seasonal (S2S) forecasts has been steadily increasing in recent years, primarily driven by certain industries, such as water management, agriculture, financial markets, energy, transportation, commerce, tourism and insurance, etc., to prepare for and reduce risk from damaging meteorological events well in advance. In 2016, the National Oceanic and Atmospheric Administration (NOAA) initiated efforts to improve its capability for Weeks 3 and 4 (i.e., 15-28 days ahead) extended range forecasts. Covering the Week 3-4 extendedrange lead time will enable NOAA to provide seamless $\mathrm{S} 2 \mathrm{~S}$ forecasts to the public for protecting life and property.

Numerical forecasts on the Week 3-4 time scale are relatively new and are some of the most challenging and difficult to make. Past forecast efforts have focused on the short-term weather forecasts out to at most 7-10 days, operational short-term climate outlooks from 6-10 days and 814 days, and months-long integrations out to several seasons. There is a clear forecast gap around Week 3 and 4 . This is because current numerical weather models perform well up to about seven days in advance, and climate outlooks become more reliable as the time horizon extends from months to seasons. Subseasonal (e.g., Week 3 4) forecasts are a middle ground, where the memory of the initial conditions that impact short-term weather is diminished after 7-10 days, while the impact of monthly and seasonal factors such as the state of El Niño, soil moisture, snow and sea ice, along with others, is not yet well established for subseasonal forecasts. Sharma et al. (2017) and Pan et al. (2019) studied precipitation forecasts in the eastern US and West Coast from short to extended range and found the current state-of-the-art models provide little useful forecast skill beyond week 1-2. Numerical forecast of the atmospheric rivers, atmospheric blocking, and tropical cyclones showed similar results (Wick et al. 2013, Nayak et al. 2014, Nardi et al. 2018, Zhong et al. 2018). Modulation of some low- 
47 frequency modes, such as the Madden-Julian oscillation (MJO), quasi-biennial oscillation (QBO),

48 and sea surface temperature (SST) suggests potential predictability for subseasonal forecasts

49 (Johnson et al. 2014, DelSole et al. 2017, Vigaud et al. 2018, Baggett et al. 2018, Mundhenk et al.

50 2018, Wang et al. 2018, Jenney et al. 2019). The Subseasonal Experiment (SubX), a research-to-

51 operations project launched recently, provides a comprehensive research infrastructure for

52 developing better S2S forecasts (Pegion et al. 2019).

53 Numerical weather and climate forecast models have been improving continuously during the

54 last several decades (Warner 2011 and Bauer et al. 2015). However, forecasts from direct

55 dynamical model outputs still suffer from large forecast errors with lead time increasing due to the

56 deficiency of model physics, errors in initial and boundary conditions, and other reasons. Therefore,

57 various dynamical model post-processing strategies are developed to remove forecast biases and

58 errors, and to nudge model predictions toward observations, before forecasts are issued to the 59 public.

60 Linear statistical post-processing methods show some success in improving direct model 61 prediction skill. One of those techniques is the model output statistics (MOS), which relates 62 observed weather elements (predictands) to appropriate model forecast variables (predictors) via 63 a statistical approach (e.g., multiple linear regression, MLR). MOS provides a tool for forecasters 64 to objectively interpret numerical model output, quantifying uncertainties, remove biases, derive 65 forecast variables not directly available from numerical forecast models, and provide improved 66 weather forecast guidance. It is used routinely in different operational centers worldwide (Glahn 67 and Lowry 1972, Klein and Glahn 1974, Wilson and Vallee 2002, 2003; Glahn et al. 2009, Gneiting 68 2014). However, the linear approach has some limitations, such as the huge number (millions) of 69 MOS forecast equations trained point-wise for different variables over different sites, projection 
70 times, and weather regimes. Moreover, with increasing lead time, the relationship between

71 predictands and predictors may be more nonlinear. This is even truer for the extended range

72 forecasts, such as the Week 3-4 forecasts.

73 In recent years, the great advances in machine learning (ML) in different fields have received

74 much attention, due to the invention of more flexible and sophisticated ML methodologies and 75 also the availability of larger datasets (i.e., "big data") for exploring challenging issues

76 (Schmidhuber 2015, LeCun et al. 2015). ML technology has been developed to work with "big

77 data" across a variety of disciplines, and impacts almost every aspect of modern society from 78 automation, classification, analysis, to detection. Modern ML (e.g., deep learning) techniques

79 allow computational models to learn representations of large data sets with multiple levels of 80 abstraction. Using a training algorithm, ML methods allow for identifying and modeling of more 81 complicated relationships between variables that are not limited by linearity with a given 82 optimization procedures.

83 Different ML techniques have been used to extract useful information and insights, and find 84 the "known unknowns" from "big data" to solve the more challenging issues and make more 85 accurate weather and climate forecasts. McGovern et al. (2017) showed that using Artificial 86 Intelligence (AI) (e.g., decision-tree-based methods) can improve high-impact weather 87 forecasting. Totz et al. (2017) used a cluster analysis for winter season precipitation anomaly 88 outlooks, which outperforms both dynamical forecast models and a canonical correlation analysis 89 based method. Cohen et al. (2019) showed ML techniques are far more powerful at mining data 90 and recognizing patterns, and may be appropriate for sub-seasonal to seasonal (S2S) predictions.

91 Neural Networks (NN) are one of the most useful methods used in ML technologies. Modern NNs 92 are able to learn high-level representations of a broad class of patterns from large datasets, and are 
93 very good at discovering intricate structures hidden within high-dimensional big data.

94 Krasnopolsky et al. $(2012,2013)$ showed that neural networks can be used to improve daily (lead

95 time of 24-hour) precipitation forecast and in many other applications in the Earth System. Liu et 96 al.(2016) used deep convolution neural networks to detect extreme weather (e.g., tropical cyclones

97 and atmospheric river) in climate data. Rasp et al. (2018) demonstrated that neural network 98 approaches can significantly outperform traditional state-of-the-art post-processing methods for 2-

$99 \mathrm{~m}$ temperature forecasts at lead time of 48-hour while being computationally affordable. NN 100 techniques have a number of advantages. Their flexible and user-friendly algorithms can be used 101 to simulate arbitrary nonlinear relationships. NN techniques can also more easily handle a large 102 numbers of predictors / predictands and may help to discover complex nonlinear interconnections 103 between predictors and predictands from large datasets.

104 So far, the daily Week $3 \sim 4$ forecast skill from direct dynamical forecast models is much 105 lower than that of the short range forecasts, such as 1 7 days and the Week 1 2 forecasts. In this 106 paper some NN architectures that are more beneficial for using model-derived fields are proposed. 107 These NNs will be used to explore and evaluate their capability to improve the Week 3-4 108 precipitation and 2-meter air temperature forecasts. The rest of this paper is organized as follows: 109 The dataset used for the NN training/testing and detailed NN methodology used in this study is 110 highlighted in section 2. The NN check, optimal hidden neurons, data representation, and analysis 111 of the Week 3-4 model forecast errors are described in section 3. The NN forecast analysis and 112 evaluation are presented in section 4, and conclusions and discussions are given in section 5.

113 2. Data and Methodology

$114 \quad 2.1$ Data for $\mathrm{NN}$ training and validation 
The datasets used for the $\mathrm{NN}$ training and testing consist of daily paired predictor and

116

117 118

119

120

121

122

123

124

125

126

127

128

129

130

131

132

133

134

135

136

137

predictand variables. The dataset for the predictors used here includes the daily bias corrected Week 3 4 lead time forecast for total precipitation (P), mean 2-meter air temperature (T2m), and 500-hPa height (Z500), and some others, which are obtained from the NOAA Climate Forecast System (CFS) (Saha et al. 2006, 2014) for the period Jan. 01, 1999 to Dec. 31, 2018. Since bias correction (by removing differences between model climatology and observed climatology) is one of the easiest and most effective ways to improve the raw model forecasts, one of the goals of this study is to see if the method introduced here can further improve the bias corrected CFSv2 forecasts. The data domain used here covers the Coterminous US (CONUS) only. The data has been re-gridded to $1 \mathrm{x} 1$ degree spatial resolution, 9 selected vertical levels (pressure: 1000, 850, $700,500,300,200,100,50$ and 10 ) and is on a daily temporal resolution initialized at 4 different times (00, 06, 12 and 18 UTC) per day. Other predictors are also used, including: daily P, T2m and Z500 climatologies, latitudes, longitudes, elevations, station ID, $\sin (\tau)$ and $\cos (\tau)$ where $\tau=$ $\frac{2 \pi}{365} t$ and $t$ is the day of the year, all on the same spatial-temporal resolutions. These auxiliary predictors are also commonly used in the MOS and other NN systems.

The datasets used for corresponding target variables (predictands) include the daily observed P from the gauge-based daily CPC Unified Precipitation Analysis, the observed daily T2m from the Global Telecommunications System (GTS) based daily 2-meter temperature analysis (Chen et al. 2008, Shi personal communication, Fan et al. 2008). Both daily observed P and T2m are converted to two-weekly total and two-weekly means, and re-gridded to the same spatial-temporal resolutions as the above predictors.

The above twenty years of daily paired (predictors and predictands) datasets have 7305 daily records and can be split into two parts, the first part (about 6575 daily records, from Jan.01,1999 
138 139 to Dec.31,2016) was used for training and the remaining part (about 730 daily records, from Jan.01, 2017 to Dec.31, 2018) was used for validation (independent forecast test). Three different k-fold cross-validation tests are also performed to verify the NN generalization in different periods.

\subsection{Methodology}

\section{a. Formulation of the problem}

Usually the statistical post processing of model output is based on the reasonable assumption that there is a relationship between target variables / predictands (e.g., observed weather and climate elements) and input variables / predictors (e.g., the corresponding forecast variables of numerical prediction model). In a generic symbolic way, this relationship can be represented as:

$$
Z=M(X) ; \quad X \in \mathfrak{R}^{n}, Z \in \mathfrak{R}^{m}
$$

where $X$ is an input vector composed of model forecast variables or predictors, $Z$ is an output vector composed of observed meteorological elements or predictands, $n$ is the dimensionality of the vector $X$ (or input space), and $m$ is the dimensionality of the vector $Z$ (or output space). $M$ denotes the mapping (relationship between the two vectors) that relates vectors $X$ and $Z$. In a particular case when a single predictand is considered, the mapping Eq. (1) turns into a single valued function of multiple variables. This function/mapping is expected to be a complex nonlinear function.

Since both model forecast variables (predictors) and observations (predictands) contain errors in their data representations due to model deficiency, noise, uncertainty in initial and boundary conditions, and limited spatial and temporal resolutions, etc., a statistical approximation of the mapping Eq.(1) can be written as:

$$
Y=M_{S}(X)
$$


159 Here the vector $Y$ can be considered as an estimated predictand vector based on model variables $160 X$, while $M_{\mathrm{s}}$ is a statistical approximation for the mapping $M$ in $\mathrm{Eq}(1)$. In the majority of modern 161 MOS systems a single valued and pointwise MLR is used as the method of statistical 162 approximation. In this case, the mapping Eq. (2) can be represented by a system of $m$ independent 163 linear regression equations:

$$
y_{q}=a_{q 0}+\sum_{j=1}^{n} a_{q j} \cdot x_{j} ; \quad q=1, \cdots, m
$$

165

166

167

168

169

170

171

172

173

174

175

176

177

178

179

180

181

The coefficients $a_{q j}$ of various equations of the system (3) are different and usually calculated for each equation (for each corrected model variable, $y_{q}$ ) individually and independently.

The linear regression approach Eq. (3) has three major disadvantages. First, the essentially nonlinear relationship/mapping Eq. (2) is approximated by linear dependencies in Eq. (3), which loses nonlinear components of the relationship between input vector and output vector. Second, the linear approach, as designed in most MOS procedures, does not consider the co-variability between output variables (e.g., the observed two week total P and mean T2m here), whereas the nonlinear relationship/mapping Eq. (2) can take into account the relationships between different observed weather elements (components of vector $Y$ ). Third, the approximation Eq. (3) splits the vector $Y\left(e . g ., \mathrm{P}, \mathrm{T} 2 \mathrm{~m}\right.$, wind and other variables) into single components, $y_{q}$, that are usually treated not only individually and independently, but also location by location (i.e., point by point), thus losing the spatial dependency (or pattern relationship). Therefore, the approach Eq. (3), by definition, does not completely use relationships and correlations (or consistency constraints) offered by the observed data. It also does not use the pattern relationships (or space dependency) offered by the big datasets.

In the following sections, it will be shown that the $\mathrm{NN}$ approach allows users not only to address the aforementioned important problems and to improve the approximation, but also greatly 
182 reduces the number of approximation equations which improves training efficiency at the same 183 time.

\section{b. NN emulation for the linear mapping}

The NN techniques are generic, accurate, flexible and convenient mathematical / statistical models that can enable users to emulate / approximate different complicated nonlinear input / output relationships, by using statistical ML algorithms (Krasnopolsky 2013). NN can be applied to any problem that can be formulated as a mapping (input vector $v s$. output vector relationship). The simplest NN approximations use a family of analytical functions such as:

$$
y_{q}=N N(X, \boldsymbol{a}, \boldsymbol{b})=a_{q 0}+\sum_{j=1}^{k} a_{q j} \cdot f_{j} ; \quad q=1,2, \ldots, m
$$

where

$$
f_{j}=F\left(b_{j 0}+\sum_{i=1}^{n} b_{j i} \cdot x_{i}\right)=\tanh \left(b_{j 0}+\sum_{i=1}^{n} b_{j i} \cdot x_{i}\right)
$$

Here, $x_{i}$ and $y_{q}$ are components of the input and output vectors $X$ and $Y$, respectively, vector $\boldsymbol{a}$ and vector $\boldsymbol{b}$ are the NN weights, $n$ and $m$ are the number of inputs and outputs respectively, and $k$ is the number of nonlinear basis activation functions $f_{j}$ (or hidden neurons). The hyperbolic tangent is used as an activation function (Hornik 1991, 1993). Other activation functions can be used depending on the problem at hand (Liu et al. 2016, McGovern et al. 2017, Rasp et al. 2018). Eq. (4) is a mapping, that can approximate any continuous or almost continuous (with finite discontinuities) mapping (Krasnopolsky 2013). A pictographic representation of the entire NN is shown in Fig. 1 and the connections (arrows) correspond to the NN weights. The NN complexity can be quickly increased by adding variables in the input layer and / or output layer, and neurons in the hidden layer.

To find the coefficients $a_{i j}$ and $b_{i j}$ in NN Eq. $(4,5)$, an error function, $E$, is created, 


$$
E=\frac{1}{N} \sum_{t=1}^{N}\left[Z_{t}-N N\left(X_{t}\right)\right]^{2}
$$

where vector $Z_{t}$ is composed of observed weather and climate elements, vector $X_{t}$ is composed of all predictors, and $N$ is the total number of paired records included in the training data set. Then, the error function (or cost function) (6) is minimized to obtain an optimal set of all coefficients $a_{i j}$ and $b_{i j}$ via a simplified version of the procedure known as the back propagation training algorithm.

The back propagation algorithm searches for the minimum of the error function in weight space through a simplified version of the steepest (gradient) descent method. It partitions the final total cost to each of the single neurons in the network and repeatedly adjusts the weights of neurons whose cost is high, and back propagates the error through the entire network from the output to its inputs.

It is noteworthy, that all NN outputs, $y_{q}$, are included in the same error function (6) and are trained simultaneously using all observed weather variables included in the output vector $Z_{i}$. Therefore, during the training, in addition to diminishing the difference between the model variables and corresponding observations, the $\mathrm{NN}$ also learns the statistical patterns and relationships between the model and observed meteorological variables, as well as nonlinear dependencies between variables included in the input vector $X$ and in the output vector $Z$ in the training dataset. These learned patterns and relationships are used by $\mathrm{NN}$ to correct the output of the numerical prediction model.

When the trained NN is applied to new data, all Eqs. (4) are evaluated simultaneously using the same coefficients $a_{i j}$ and $b_{i j}$ for all outputs. Thus, from an algorithmic point of view, all $\mathrm{m}$ Eqs. (4) are one object - a mapping; whereas the MLR approach (3) is a set of independent functions. At first sight, Eqs. (3) and Eqs. (4) look similar, however, three important differences should be emphasized. In Eq. (4): 
(a) The relationship between $x_{i}$ and $y_{q}$ is nonlinear when the activation function is nonlinear, such as tanh; therefore, the NN approximation (4) is capable of approximating both the linear and nonlinear components of the mapping (4) (Krasnopolsky 2013).

(b) The NN approximation (4) can approximate not only pattern relationships and correlations between input variables and output variables, but also the relationship (or co-variability) between different observed variables offered by the observed data included in the NN output vector $\mathrm{Z}$.

(c) By including multiple variables in the $\mathrm{NN}$ output vector at multiple locations, the $\mathrm{NN}$ approach (4) also allows the algorithm to significantly reduce the maintenance burden on the $\mathrm{NN}$ equations by generating all weights in one training cycle and storing them in one array file. In contrast, the MLR (e.g., MOS) approach in Eq. (3) usually consists of several thousand to several million individual and independent equations.

\section{c. Design NN architectures}

Effective training the NN system requires not only designing the NN architecture with faithful representation of training data, but also careful tuning of the parameters, such as the number of neurons, learning rate, regularization, and adding appropriate auxiliary variables in order to achieve more optimal results, avoid overfitting, and achieve better generalization (Krasnopolsky 2007, 2013, Rasp et al. 2018, Fan et al. 2019). In this study, three different NN architectures are designed or configured as follows:

(a) $\mathrm{NN}-1$, which can produce one corrected CFS variable (e.g., P or T2m) at one location (grid point) like the MLR. This pointwise NN setting has an architecture n:K:1 (n inputs at one location: K hidden neurons: 1 output at 1 location). 
249 (b) NN-S, which can produce one and/or several corrected CFS variables (e.g., P and/or T2m) at one or several locations (grid points) simultaneously. One NN-S training can replace two or more MLR equations needed to reach the same goal in the traditional MLR approach. This NN setting has an architecture n:K:m (n inputs from one or several locations: K hidden neurons: m outputs at one or several locations). NN-S can be treated as a small regionalized architecture by setting n:K:m (n inputs from a small region: K hidden neurons: $\mathrm{m}$ outputs in a small region that is not necessarily the same as the input region).

(c) NN-A, which can produce one and/or several corrected CFS variables (e.g., P and/or T2m or more variables) for the entire forecast domain simultaneously. In this case, both $\boldsymbol{X}_{i}$ and $\boldsymbol{Y}_{q}$ in Fig.1 are vector variables. This NN setting has an architecture L:K:M (L inputs from all available predictors over all input locations: $\mathrm{K}$ hidden neurons: $\mathrm{M}$ outputs from all available predictands over the all forecast domain). Here $\mathrm{L}$ and $\mathrm{M}$ are not necessarily in the same domain. In principle, one NN-A training could replace several thousand MLR equations needed to reach the same goal in the traditional MLR approach. NN-A not only benefits from the flexible NN algorithms, but also takes full advantage of the available big data.

It should be emphasized that the NN-A architecture allows the algorithm to account for both nonlinear relationships among input and output variables, and for the spatial dependency and the co-variability among the predictors and predictands by training different predictor and predictand variables over the entire forecast domain simultaneously. During the NN-A training, the NN algorithm tries to minimize the differences between all predictors and predictands at all input and output locations simultaneously to obtain an optimal set of the NN weighting coefficients for all locations. The statistical patterns and relationships learned during the NN training processes are 
272 then used by the NN to make the corrected forecasts for each locations. Doing it all at once in an

$273 \mathrm{NN}$ method does not mean regional differences are neglected.

It should also be noted that the complexity of the $\mathrm{NN}$ approximation is partly controlled by

275 the number of hidden neurons, K. The more complicated the mapping, the more hidden neurons K

276 are required. However, there is always a trade-off between the desired mapping accuracy and

277 complexity of the NN emulation. The number K should be carefully controlled and kept to a

278 minimum in order to avoid overfitting and to allow a smooth and accurate mapping. The weight

279 initialization method (Nguyen et al. 1990) is used for reducing the effects of overfitting and

280 achieving better generalization. The NN weights can be updated inexpensively on a daily basis in

281 real-time, through a sequential training approach that works with the training data arriving in real-

282 time (record by record).

3. NN Check, Optimization, Data Representation, and Predictability Analysis

\subsection{NN sanity check}

In order to evaluate the accuracy of the NN approximation and also the applicability of NN

software used, the NN-1 was trained at several randomly selected locations to approximate the

287 identical mapping:

$$
X=M(X)
$$

289 where $X$ could be any predictor and predictand variable. If the $\mathrm{NN}$ is working properly, a mapping 290 performed between a variable $X$ and itself should return the variable $X$. Figure 2 shows the 291 independent Week 3 4 precipitation mapping from the NN-1 approximation and the observed two 292 week total precipitation in the same period from one of several randomly selected locations 293 (Tucson, AZ). The NN-1 training period is from January 01, 1999 to December 31, 2015. The 294 experiment indicates that the NN algorithm can almost perfectly reproduce the observed 
295

296

297

298

299

300

301

302

303

304

305

306

307

308

309

310

311

312

313

314

315

316

317

precipitation for the independent forecast period from March 01, 2016 to February 28, 2017. The difference between the above $\mathrm{NN}-1$ and the observation varies between $-0.2 \mathrm{~mm}$ and $0.4 \mathrm{~mm}$. Similar mapping also has been done on the CFS Week 3 4 forecast precipitation with similar results. The NN-1 also can reproduce the nosier CFS model forecasts very well with slightly higher mapping errors for reasons noted below in section 3.3.

\subsection{Optimal number of hidden neurons}

The complexity of the NN mapping can be controlled by varying the number of the NN hidden neurons. To evaluate the optimal size $(k)$ of the hidden neurons in equation (4) for the NN Week 3 4 $\mathrm{P}$ and T2m forecasts, some criteria, such as the root mean square error (RMSE), Bias, Correlation, Scatter, Skewness, and others are used together to select the optimal number of hidden neurons. A set of fourteen NN-S (9 neighbor points used here) are trained with varying $k$ from 1 to 14. The results based on the widely used RMSE are shown in Fig.3 (a) with the independent NN forecasts. For the precipitation forecast on a pointwise basis, $k=3$ is the optimal number of $\mathrm{NN}$ hidden neurons. Under the chosen NN-S setting, using more neurons $(k>3)$ does not reduce the forecast error, probably because the NN-S starts to fit more noise from the data. The results from NN-1 are very similar to those from the above NN-S settings. When compared with the benchmark MLR method with the same predictors, both NN-1 and NN-S do a better job at predicting the observed precipitation. However, in terms of optimal hidden neurons, the mapping from both NN1 and NN-S is not strongly nonlinear (i.e., only a small number $k$ can be used beneficially).

In order to evaluate the optimal number of hidden neurons in Eq. 4 for the NN-A, another set of fifteen NN-A tests $k$ varying from 10 to 220 has been conducted. The mean forecast RMSE derived from the Week 3 4 forecast $\mathrm{P}$ and $\mathrm{T} 2 \mathrm{~m}$ using independent testing data set is shown in Fig.3 (b). The results indicate that if separately forecasting $\mathrm{P}$ or T2m, $k=120$ is near the optimal 
number of hidden neurons. In contrast, forecasting $\mathrm{P}$ and $\mathrm{T} 2 \mathrm{~m}$ together requires $\mathrm{k}=200$ hidden neurons for optimal results. This indicates that the NN-A architecture with more than 100 hidden neurons is significantly more nonlinear than NN-1 and NN-S architectures with a far lower set of hidden neurons $(\sim 2-3)$. In other words, with NN-A the nonlinear and pattern-wise corrections for the Week $3 \sim 4$ forecasts of both P and T2m over the entire forecast domain (CONUS) is much more ambitious and potentially beneficial than the point-wise correction for just a single location or several neighboring sites. Therefore, the NN-A mapping, which is designed to take advantage of the flexible $\mathrm{NN}$ algorithm and big datasets and to do more sophisticated pattern-wise corrections, presents much more non-linear features, as expressed in terms of the optimal number of hidden neurons. In general, the computational cost increases linearly with hidden neurons used.

\subsection{Data representation}

It is important to understand the characteristics of the data being analyzed because it will inform choice in the $\mathrm{NN}$ architectures. When looking at the time series of the daily CFS Week 3 4 forecast total precipitation and its corresponding observed total precipitation, two significant differences emerge:

(1) The observed total precipitation (e.g., light-blue solid curves in Fig. 4) is smoother than its corresponding CFS Week 3 4 forecast total precipitation (black dot curves in Fig. 4). This is because each of the daily observed 2-week total precipitation has a 13-day overlap of data on its adjacent date. However, for each of the daily CFS Week $3 \sim 4$ forecast total precipitation, the model forecasts do have such 13-day overlap in terms of dates, but they come from different initializations. Due to forecast error growth, the CFS data is noisier compared to observations.

(2) The trajectories of the daily CFS Week 3-4 forecast total precipitation at the same location, but initialized at 4 different initial times (00Z, $06 \mathrm{Z}, 12 \mathrm{Z}$ or $18 \mathrm{Z}$ on each day), can be very different 
341 after 4 weeks of model integration. However, how to address the above issues in training datasets

342 properly is crucial for improving the $\mathrm{NN}$ training.

343 To minimize impacts related to the above two issues, the empirical orthogonal function (EOF) 344 analysis was used to explore the spatial-temporal variations of the bias corrected CFS Week 3 4 345 forecast P and T2m in the period covering January 01, 1999 to Dec 31, 2018 from 4 different initial 346 times. The encouraging results indicate that the leading EOF patterns and the variations of their 347 corresponding time series are quite similar from the four different initial times $(00,06,12 \& 18$ 348 UTC). Fig. 5 depicts the first 4 leading EOF patterns and their corresponding time series from the 349 CFS Week 3 4 ensemble mean total precipitation (averaged from 00Z, 06Z, 12Z and 18Z), which 350 are similar to results from the individual CFS Week 3 4 total precipitation forecasts initialized at 351 00Z, 06Z, 12Z and 18Z. The spatial patterns of the leading EOF modes are relatively large-scale 352 and the temporal variations are dominated by annual and semiannual cycles. The first 4 modes 353 account for about $57 \%$ of the total variance from the ensemble mean forecasts, but only about $44 \%$ 354 of the total variance from individual members.

The EOF analysis was applied to the corresponding observed 2-week total precipitation. The 356 first 4 leading EOF modes account for about $42 \%$ of the total variance from the observed 2 week 357 total precipitation. It shows that at large scales (the first 4 leading EOF patterns) the CFS Week $3583 \sim 4$ forecast total precipitation bears many similarities with observational data. However, the 359 corresponding time series from the observational data are noisier, except for the first leading EOF, 360 the variation of its time series is also dominated by a very strong annual cycle.

The same EOF analysis was also applied to the CFS Week 3 4 ensemble mean forecast T2m 362 and its corresponding observed 2-week mean T2m. The results (not shown) reveal that the leading 363 EOF spatial patterns from the CFS Week 3 4 forecast T2m are dominated by large scale patterns 
and are remarkably similar to those from the observational data. However, the amplitudes and timing are main issues for the CFS Week $3 \sim 4$ forecasts. The first 4 leading EOF modes account for $84 \%$ of the total variance for the CFS Week $3 \sim 4$ ensemble mean T2m forecasts and $78 \%$ of the total variance for the observational data. This suggests that the structures of the $\mathrm{T} 2 \mathrm{~m}$ are simpler than the P.

The above results suggest that the CFS is comparatively better at predicting large-scale patterns and low frequency variations in the observed $\mathrm{P}$ and $\mathrm{T} 2 \mathrm{~m}$ than at capturing fine scale variations of those highly parameterized and unresolved physical processes. These results indicate important suggestions in the $\mathrm{NN}$ training processes:

(i) Using more reliable and robust large-scale pattern information in the NN predictors (e.g. the CFS forecast P, T2m and Z500) may prove to be more beneficial for the $\mathrm{NN}$ forecasts.

(ii) Using ensemble means (average from 00, 06, 12 \& 18 UTC) may further improve data representation, because ensemble mean not only smooths spatial-temporal noise in the input data, but also increases the percentage representation (explanation) of the total variance of the data.

\subsection{Analysis of the CFS Week 3-4 P and T2m Forecast Errors}

The similar EOF analysis was also applied to the bias-corrected CFS Week 3 4 ensemble mean P and T2m forecast errors (i.e., forecast minus observation). Moreover, such an EOF analysis can also provide insight into limits of CFS Week 3 4 P and T2m forecasts (in other words, what do the CFS Week 3-4 forecast errors look like and to what extent can the errors be removed?). Ideally, if the forecast errors are either constant or vary regularly, then nearly all errors can be removed easily. If the forecast errors are characterized by large-scale spatial pattern and low frequency temporal variations, then at least part of the errors can be corrected in most cases. The worst scenario is if forecast errors are white noise-like. In that scenario, there is no way the forecast 
errors can be corrected or removed, no matter what methods are used.

The results (Fig. 6) reveal that in general these leading EOF patterns from the bias corrected CFS Week 3 4 ensemble mean P forecast errors are relatively large-scale patterns and feature some low-frequency variations (e.g., annual cycle), but are much nosier compared with the time series in Fig. 5. These forecast errors are caused by the model deficiency, errors in initial and boundary conditions, definition differences of the model forecast vs. observed variables, and the nature of predictands. They may be partly removable by some post-processing techniques. For example, the first and second leading forecast error modes feature relatively large-scale patterns and are dominated by the annual cycle. This means that the CFS does not produce a satisfactory forecast for the observed annual cycle in precipitation over CONUS in terms of the amplitudes and phases. The good news is that usually part of these climate-like forecast errors (or climate biases) can be easily removed by some bias correction methods (Fan and van den Dool 2011).

It should be mentioned that the above forecast errors from the first 4 leading EOF modes only account for about $34 \%$ of the total variance from the Week $3 \sim 4$ ensemble mean P forecast errors, meaning limited opportunity for forecast improvement over the CFS week 3 4 P forecasts. Not all of these forecast errors are correctable (or removable). In general, the higher the EOF leading mode, the smaller the scale in spatial pattern and the noisier in temporal variation. Usually these small-scale and high-frequency forecast errors are even more difficult to remove. To some extent, they may reflect the prediction limits for the CFS Week 3 4 precipitation forecasts.

For the bias corrected CFS Week 3 4 ensemble mean T2m forecast errors, the most dominant (i.e., the first 4 leading EOF) forecast error patterns (Fig. 7) show large-scale spatial patterns very similar to the forecasts and observations individually. The corresponding time series also feature some low-frequency (e.g., annual cycle) variations. The first 4 leading EOF modes account for 
410

411

412

413

414

415

416

417

418

419

420

421

422

423

424

425

426

427

428

429

430

431

432

$78 \%$ of the total variance for the forecast errors, much higher than the forecast $\mathrm{P}$ and therefore potentially more predictable than the P. This means that a large part of the $\mathrm{T} 2 \mathrm{~m}$ forecast errors can be represented by just a few leading EOF modes. These climate bias-like forecast errors indicate that the CFS is very good at forecasting the Week $3 \sim 4 \mathrm{~T} 2 \mathrm{~m}$ spatial patterns, but has problems forecasting their amplitudes and timing. Compared with the forecast P errors, these $\mathrm{T} 2 \mathrm{~m}$ forecast errors should be comparatively easier to remove in general. However, because the time-series of the first 4 leading EOF modes are as noisy as is the case for forecast P errors, it may still be difficult to remove these $\mathrm{T} 2 \mathrm{~m}$ forecast errors. Some features of the above forecast errors are also true for the short-range weather forecasts from day-1 up to Week 2 in some forecast systems, such as NCEP Global Forecast Systems (GFS) (Fan et al. 2011, 2015).

\section{NN Week 3 4 P and T2m forecasts}

\subsection{Forecasts from different NN architectures}

There is considerable need for skillful Week 3-4 forecasts. However, forecasting for this time scale is one of toughest areas and prediction skills are very low in general. One open question to be explored here is if the ML (e.g., the nonlinear NN systems used here) techniques with the bias corrected CFS predictors as input can outperform the bias corrected CFS P and T2m forecasts in Week 3-4 time scale and the benchmark MLR tools with the same inputs as the NN systems.

The daily time series of the Week 3 4 P from the observational data, the bias corrected CFS forecast, the MLR forecast, and the NN forecast for three randomly selected locations are also shown in Fig. 4 above. Overall, the results from the NN forecasts are slightly better than the results from the MLR method. In general, both methods beat the climatology forecasts, but not by much. The forecast skill (in terms of the RMSE) for the Week 3-4 precipitation is still quite low. The results also indicate that the resulting Week $3 \sim 4 \mathrm{NN}$ precipitation forecasts by using the ensemble 
mean from 4 initial times $(00 \mathrm{Z}, 06 \mathrm{Z}, 12 \mathrm{Z}$ and $18 \mathrm{Z})$ are in general better than the $\mathrm{NN}$ forecasts by using the CFS forecasts from an individual ensemble member. Similarly, the Week 3-4 forecasts by using NN-A generally outperform those from the NN-1 or NN-S, since NN-1 and NN-S settings do not fully take the benefits offered by the NN algorithms and big data by only working on very small portions of data at a given time.

As mentioned earlier, the NN-A setting can take advantage of the flexible $\mathrm{NN}$ algorithm that accounts for complicated linear and non-linear relationships, spatial dependency, and covariability among predictors and predictands. The NN-A setting was explored with a variety of predictors and predictands. The results show that using observed daily P and T2m climatologies as predictors outperforms other auxiliary predictors, such as $\sin (\tau), \cos (\tau)$, latitude, longitude, elevation, station ID, etc., because all these effects are already well represented by the climatology variables. It also shows that using the same group of predictors to forecast the Week 3-4 P and $\mathrm{T} 2 \mathrm{~m}$ together (co-variability between observed $\mathrm{P}$ and $\mathrm{T} 2 \mathrm{~m}$ counted) is better than forecasting the same P and T2m separately.

In the following part of this paper, the focus will turn to the more beneficial NN-A setting by forecasting P and T2m together. The five predictors used in NN training include the CFS bias corrected ensemble mean Week 3-4 total P, anomaly T2m and Z500, and the observed P and T2m climatologies. The two predictands are observed total $\mathrm{P}$ and anomaly $\mathrm{T} 2 \mathrm{~m}$.

\subsection{Verification of the daily NN Week 3-4 P and T2m forecasts}

In this subsection, the spatial-temporal distribution of the Week 3-4 P and T2m forecast skill will be explored. Figure 8 shows that overall the root mean square error (RMSE) and anomaly correlation coefficients (AC) of the bias corrected ensemble mean CFS precipitation forecasts when adjusted by the NN-A are better than the adjusted forecast obtained from the benchmark 
456 pointwise MLR method for most locations. Here the NN-A and MLR training period is from $457 \quad 01 / 01 / 1999$ to $12 / 31 / 2016$. The period of $01 / 01 / 2017$ to $12 / 31 / 2018$ is used as an independent 458 verification period. The above results indicate both the NN-A and MLR methods improve the bias 459 corrected CFS Week 3 4 precipitation forecasts, especially the forecast skill in various parts of the 460 western CONUS are encourging (AC over 0.4 or 0.5 ). However, some degradation is also seen in 461 limited areas, such as near the western coast. In general, the NN-A forecasts show better forecast 462 skills than the MLR forecasts over most locations in term of both the RMSE and AC, with the AC 463 improvement more robust. This may also indicate that accounting for the non-linear relationship 464 between the predictors and predictands, as well as making use of co-linearity plays an important 465 role in precipitation forecasting.

466 As Figure 8, Figure 9 shows the Week 3 4 T2m forecast skills from the bias corrected CFS, 467 the MLR, and the NN-A methods. Both the NN-A and the MLR are able to reduce the CFS forecast 468 errors in terms of the RMSE, although not as much. The performance of the NN-A is slightly better 469 than the MLR method, in terms of the RMSE forecast skill. However, in terms of the AC forecast 470 skill it is encouraging that the NN-A method is significantly better than the MLR method in most 471 places, except some degradation in limited areas. Again, this may indicate that the non-linear 472 relationship plays an important role between the predictors and predictands at improving the Week $473 \quad 3-4 \mathrm{~T} 2 \mathrm{~m}$ forecasting.

\subsection{Three K-fold cross-validations}

In this subsection, three multi-year daily NN Week 3-4 P and T2m (independent) forecast experiments were conducted to further explore whether the Week 3-4 P and T2m forecast

477 improvement from the ML (e.g., the nonlinear NN systems used here) technologies are robust, 478 reliable, and meaningful when compared with the bias corrected CFS Week 3 4 forecasts. Three 
$\mathrm{k}$-fold cross-validation tests were performed as follows:

Test1: Remove 3 years of daily paired data from a 20-year period (1999-2018) of daily pooled data sequentially and yearly, then use the middle year only as the independent forecast (testing) dataset, with the remaining 17 years daily paired data employed as training dataset. For the year 1999 and 2018, only two years daily data is removed, with the far side year (i.e., 1999 or 2018) used only as an independent forecast (testing) dataset, and the remaining 18 years daily data used as the primary training dataset. The above procedure was repeated yearly for 20 times so that the independent NN-A experiments were performed for every year from 1999 to 2018.

Test2: Remove one year of daily paired data from a 20-year period (1999-2018) of daily pooled data sequentially and yearly, taking these as the independent forecast (testing) dataset and the remaining 19 years daily paired data as the training dataset. The above procedure was repeated yearly for 20 times. Therefore, another 20 yearly independent NN-A experiments were performed from 1999 to 2018.

Test3: Remove 60 days of daily paired data (each in 2012-2018) from a 20-year period (19992018) of daily pooled data sequentially as the independent forecast (testing) dataset, using the remaining 19 plus years of daily paired data as the training dataset. A total of $42 \mathrm{NN}-\mathrm{A}$ 60-day independent experiments cover the period from 2012 to 2018.

Figure 10 shows the time-series of the daily Week 3-4 forecast P and T2m spatial anomaly corrections (AC) averaged over 2012 to 2018 from (i) the NN (Test 3) independent forecasts, (ii) the NN dependent forecasts (training data covering 1999-2018, can be viewed as the upper limit of $\mathrm{NN}$ forecasts) and (iii) the bias corrected CFS forecasts. The results indicate that the NN techniques indeed can make a robust improvement for the Week 3-4 P and T2m forecasts over the bias corrected CFS forecasts. Both of the independent NN Week 3-4 P and T2m forecasts are 
502 improved over the bias corrected CFS P and T2m forecasts, with the NN Week 3-4 P forecast

503 improvement (mean AC from 0.05 to 0.21 ) being a more robust improvement across all times of

504 the year, while the NN Week 3-4 T2m forecast improvement (mean AC from 0.16 to 0.24 ) is less

505 robust than the P forecasts. The results also show that the independent NN (Test 3) Week 3-4 P and

$506 \mathrm{~T} 2 \mathrm{~m}$ forecasts have very similar tendencies as the dependent NN Week 3-4 P and T2m forecasts.

507 This indicates that sometimes the dependent NN forecast systems are more predictable than other 508 times and the independent NN forecast systems follow the same ups and downs.

509 For Test 2, the mean time-series of the daily NN Week 3-4 forecast P and T2m spatial anomaly 510 correlations closely follow the results from Test 3, with forecast skill degraded slightly (mean AC 511 from 0.21 to 0.20 for $\mathrm{P}$ and from 0.24 to 0.22 for $\mathrm{T} 2 \mathrm{~m}$ ), due to the training sample data being 512 farther away from the dependent training sample data. For Test 1, its mean time-series of the daily 513 NN Week 3-4 forecast P and T2m spatial anomaly correlations also follow the results from Test 3 514 quite well with forecast skill further degraded (mean AC from 0.21 to 0.19 for P and from 0.24 to 5150.20 for $\mathrm{T} 2 \mathrm{~m}$ ), due to the training sample data being even farther away from the dependent training 516 data. Therefore, in principle, if we can nudge the training sample (e.g., Test 3, but withhold 30 517 days data as independent test data) closer to the dependent training sample, the forecast skill should 518 be further improved when compared with Test 3.

\subsection{Comparison of different forecast methods}

Finally, when checking the overall Week 3-4 forecast performance of three (CFS, MLR and

$521 \mathrm{NN}$ ) forecasts over the multi-year verification period, both the MLR and the NN consistently beat

522 the bias corrected CFS. Of the MLR and NN forecasts, the NN forecasts significantly 523 outperformed the pointwise MLR forecasts in many respects. Figure 11 to Figure 14 depict 524 examples of the observed Week 3-4 P and T2m anomalies, together with the corresponding Week 
3-4 CFS, MLR, and NN forecast P and T2m anomalies. In these cases, the NN techniques show very encouraging and impressive ability to turn around or reverse the incorrect $\mathrm{P}$ and $\mathrm{T} 2 \mathrm{~m}$ forecast patterns seen in the bias corrected CFS forecasts. Usually the above "turn around" events can persist for several days and can happen in any season. One possible explanation for this is that model forecast spatial patterns are systematically and frequently offset in certain time frames and locations with certain P, T2m and Z500 patterns, and the NN architecture used here has the ability to allow the NN algorithm to remember what happened. Then, the NN system can determine what is (are) the best and most important forecast input(s), where these (group) points are located, and how to minimize the forecast errors in multiple dimensions for best mapping the target (predictand) points, an accomplishment that cannot be done with the traditional pointwise and spatially independent MLR method.

\section{Conclusion and Discussion}

In this study, NN techniques are used to improve the NCEP CFS Week 3 4 P and T2m forecasts, and to explore the predictability of the CFS Week 3 4 P and T2m forecasts. Benefiting from the great advances in ML in recent years, NN techniques show some advantages over traditional statistical methods such as MLR: its flexible algorithms can account for complicated linear and non-linear relationships, spatial dependency, and co-variability in predictors and predictands, and at the same time, it is able to handle big data easily and efficiently.

Knowing the datasets well and using a better data representation are very important before applying NN training. The EOF analysis indicates that the CFS is very good at predicting largescale patterns and low frequency variations in observed $\mathrm{P}$ and $\mathrm{T} 2 \mathrm{~m}$, but less so at capturing highly parameterized and unresolved processes in $\mathrm{P}$ and $\mathrm{T} 2 \mathrm{~m}$. Better data representation can also be 
547 achieved by using ensemble means to increase the explained percentage of total variance and to 548 reduce noise in the data.

The EOF analysis of the CFS Week 3 4 P and T2m forecast errors provides some insight on the extent that forecast errors are correctable. The results reveal that the spatial-temporal structures 551 of the most dominant CFS Week 3 4 forecast errors have relatively large-scale spatial patterns 552 with low frequency variations, such as the annual cycle, namely climate biases. This is also true 553 for some short range weather forecast systems from day-1 up to 2-weeks. In general, at least part 554 of these large-scale and low-frequency forcast errors are removable.

Different NN configurations are used to compare to the benchmark MLR postprocessing 556 method. By designing more beneficial NN setups, the NN-A architecture, is able to account for 557 not only nonlinear features or relationship within a given large dataset, but also spatial dependency 558 (e.g., pattern relationships) by training different predictors and predictands from the entire forecast 559 domain simultaneously. Moreover, the NN-A architecture can also account for the co-variability 560 among the predictands by training different predictands simultaneously. Together, these learned 561 statistical patterns and relationships from the NN training processes are then used to correct the 562 CFS Week 3-4 forecasts. The NN-A has the ability to extract more complicated and high-level 563 information hidden behind big data. Thus, the NN-A can perform more sophisticated forecast 564 corrections, such as reversing incorrect forecast patterns, which is impossible for the traditional 565 method like pointwise MLR.

Althrough the improvement for the Week 3 4 P and T2m is very encourging, the overall 567 forecast skill (in terms of both RMSE and AC skills) for the Week 3 4 P and T2m predictions is 568 still quite low, when compared to the Week 2 outlooks. Since the NN forecasts here critically 569 depend on the quality of the CFS forecast inputs, improving the CFS itself remains critically 
570 important to improve the Week 3 4 forecasts. Another potential way to improve the CFS Week

$571 \quad 3 \sim 4 \mathrm{P}$ and $\mathrm{T} 2 \mathrm{~m}$ forecasts is to do more detailed dynamic analyses and to consider including more

572 related predictors. Using more advanced NN architectures (e.g., deep-NNs) and more advanced

573 ML techniques could also help to improve the forecasts. Further studies can advance this capability.

575 Data Availability:

576 CFSv2 can be downloaded from NOAA NCEI (former NCDC) web site.

577 CPC daily T2m analysis: ftp://ftp.cpc.ncep.noaa.gov/precip/PEOPLE/wd52ws/global_temp/

578 CPC daily P analysis: ftp://ftp.cpc.ncep.noa.gov/precip/CPC UNI PRCP/GAUGE GLB/

579

580

Arcknowledgement: We thank Drs. Peitao Peng and Kyle MacRitchie for their constructive suggestions and insightful comments. Thanks also due to the three anonymous reviewers for their excellent comments and suggestions. This work was partly supported by NOAA OSTI program.

\section{References}

Bauer, P., A. Thorpe, and G. Brunet, 2015: The quiet revolution of numerical weather prediction.

587 Nature, 525, 47-55, https:// doi.org/10.1038/nature14956.

589 forecasts of weekly tornado and hail activity using the Madden-Julian Oscillation.J. Geophys. 590 Res., 123, 12 661-12 675, doi:10.1029/2018JD029059. 
591 Chen, M., W. Shi, P. Xie, V. B. S. Silva, V E. Kousky, R. Wayne Higgins, and J. E. Janowiak 592 (2008), Assessing objective techniques for gauge-based analyses of global daily precipitation, J.

593 Geophys. Res., 113, D04110

594 Cohen, J., D. Coumou, J. Hwang, L. Mackey, P. Orenstein, S. Totz, and E. Tziperman, 2019: 595 S2S reboot: An argument for greater inclusion of machine learning in subseasonal to seasonal 596 forecasts. WIREs Clim Change. 2019; 10:e567. https://doi.org/10.1002/wcc.567

597 DelSole, T. and L. Trenary, 2017: Predictability of Week-3-4 Average Temperature and 598 Precipitation over the Contiguous United States. J.Climate, 30, 3499-3512.

599 https://doi.org/10.1175/JCLI-D-16-0567.1

600 Fan, Y. and H. van den Dool, 2008: A global monthly land surface air temperature analysis for 601 1948-present, J. Geophys. Res., 113, D01103, doi:10.1029/2007JD008470.

602 Fan, Y. and H. van den Dool, 2011: Bias Correction and Forecast Skill of NCEP GFS Ensemble 603 Week1 and Week2 Precipitation, 2m Surface Air Temperature and Soil Moisture Forecasts.

604 Weather and Forecasting, 26, 355-370

605 Fan, Y., K. Gilbert, D. Rudack, W. Yan, S. Scallion, and P. Shafer, 2015: The Characteristics of 606 GFS MOS Temperature Forecast Guidance Errors for Past Decade.

607 https://ams.confex.com/ams/95Annual/webprogram/Paper265326.html

608 Fan, Y., C-Y, Wu, J. Gottschalck and V. Krasnopolsky 2019: Improve CFS Week 3-4

609 Precipitation and 2 Meter Air Temperature Forecasts with Neural Network Techniques.

610 https://doi.org/10.25923/ae2c-v522, 59-63. 
611 Gneiting, T., 2014: Calibration of Medium-Range Weather Forecast. ECMWF Technical

612 Memorandum No. 719

613 Glahn, H. R., and D. A. Lowry, 1972: The use of model output statistics (MOS) in objective

614 weather forecasting. J. Appl. Meteor., 11, 1203-1211.

615 Glahn, H. R., Gilbert, K., Cosgrove, R., Ruth, D. P. and Sheets, K. (2009). The gridding of MOS. Weather 616 and Forecasting, 24, 520-529.

617 Hornik, K. Approximation Capabilities of Multilayer Feedforward Network. Neural Networks, $6181991,4,251-257$

619 Hornik, K. Some new results on neural network approximation. Neural Networks, 1993, Volume 620 6, Issue 8, 1993, Pages 1069-1072; https://doi.org/10.1016/S0893-6080(09)80018-X

621 Jenney, Andrea, Kyle Nardi, Elizabeth Barnes, and David Randall, 2019: The Seasonality and

622 Regionality of MJO Impacts on North American Temperature, Geophysical Research Letters, 623 https://doi.org/10.1029/2019GL083950.

624 Johnson, N. C., Collins, D. C., Feldstein, S. B., L'Heureux, M. L., \& Riddle, E. 625 E. (2014). Skillful wintertime North American temperature forecasts out to 4 weeks based on the 626 state of ENSO and the MJO. Weather and Forecasting, 29(1), 23- 38.

627 https://doi.org/10.1175/WAF-D-13-00102.1

628 Klein, W. H. and Glahn, H. R. (1974). Forecasting local weather by means of model output 629 statistics. Bulletin of the American Meteorological Society, 55, 1217-1227.

630 Krasnopolsky, V. 2007: "Reducing Uncertainties in Neural Network Jacobians and Improving 631 Accuracy of Neural Network Emulations with NN Ensemble Approaches", Neural Networks, 20, 632 pp. 454-461. 
633 Krasnopolsky V. M. and Y. Lin, 2012: "A Neural Network Nonlinear Multimodel Ensemble to 634 Improve Precipitation Forecasts over Continental US", Advances in Meteorology, Volume 2012, 635 Article ID 649450, 11 pages, doi:10.1155/2012/649450

636 Krasnopolsky, V. 2013: "The Application of Neural Networks in the Earth System Sciences. 637 Neural Network Emulations for Complex Multidimensional Mappings", Springer, 200 pp.

638 LeCun, Y., Y. Bengio, and G. Hinton, 2015: Deep learning. Nature, 521, 436-444, 639 https://doi.org/10.1038/nature14539.

640 Liu, Y., E. Racah, J. Correa, A. Khosrowshahi, D. Lavers, K. Kunkel, M. Wehner, and W. 641 Collins, 2016: Application of deep convolutional neural networks for detecting extreme weather 642 in climate datasets. arXiv.org, https://arxiv.org/abs/1605.01156

643 McGovern, A., K. L. Elmore, D. J. Gagne, S. E. Haupt, C. D. Karstens, R. Lagerquist, T. Smith, 644 and J. K. Williams, 2017: Using artificial intelligence to improve real-time decision-making for 645 high-impact weather. Bull. Amer. Meteor. Soc., 98, 2073-2090, https://doi.org/10.1175/BAMS$646 \quad \underline{D-16-0123.1}$

647 Mundhenk, B., E. Barnes, E. Maloney, and C. Baggett, 2018: Skillful empirical subseasonal pre648 diction of landfalling atmospheric river activity using the Madden-Julian oscillation and quasi649 biennial oscillation. Nature Clim. and Atmos. Sci.,1, doi:10.1038/s41612-017-0008-2.

650 Nardi, K., E. Barnes, and F. Ralph, 2018: Assessment of numerical weather prediction model 651 reforecasts of the occurrence, intensity, and location of atmospheric rivers along the west coast 652 of North America. Mon. Wea. Rev., 146, 3343-3362, doi:10.1175/MWR-D-18-0060.1. 
653 Nayak, M., G. Villarini, and D. Lavers, 2014: On the skill of numerical weather prediction 654 models to forecast atmospheric rivers over the central United States. Geophys. Res. Lett., 41, 655 4354-4362, doi:10.1002/2014GL060299.

656 Nguyen D and B. Widrow, 1990: Improving the learning speed of 2-layer neural networks by 657 choosing initial values of the adaptive weights. Proc of International Joint Conference of Neural 658 Networks, June 17-21, San Diego, CA, USA, 3: 21-26

659 Pan, B., K. Hsu, A. AghaKouchak, S. Sorooshian, and W. Higgins, 2019: Precipitation 660 prediction skill for the west coast United States: From short to extended range. J. Climate, 32, 661 161-182, doi:10.1175/JCLI-D-18-0355.1.

662 Pegion, K. and Coauthors, 2019: The Subseasonal Experiment (SubX): A Multimodel 663 Subseasonal Prediction Experiment. Bull. Amer. Meteor. Soc., 100, 2043-2060, 664 https://doi.org/10.1175/BAMS-D-18-0270.1

665 Rasp, S and S. Lerch, 2018: Neural Network for Postprocessing Ensemble Weather Forecasts. 666 Mon. Wea. Rev., 146, 3885-3900, https://journals.ametsoc.org/doi/pdf/10.1175/MWR-D-18$667 \quad \underline{0187.1}$

668 Saha, S. and Coauthors, 2006: The NCEP Climate Forecast System. J. Climate, 19, 3483-3517.

669 Saha, S. and Coauthors, 2014: The NCEP Climate Forecast System Version 2. J. Climate, 27, 670 2185-2208. doi: http://dx.doi.org/10.1175/JCLI-D-12-00823.1

671 Schmidhuber, J., 2015: Deep learning in neural networks: An overview. Neural Networks, 61, 672 85-117, https://doi.org/10.1016/ j.neunet.2014.09.003. 
673 Sharma, S., and Coauthors, 2017: Eastern U.S. verification of ensemble precipitation forecasts.

674 Wea. Forecasting, 32, 117-139, doi:10.1175/WAF-D-16-0094.1.

675 Totz, S., Tziperman, E., Coumou, D., Pfeiffer, K., \& Cohen, J. 2017: Winter precipitation 676 forecast in the European and Mediterranean regions using cluster analysis. Geophysical Research

677 Letters, 44, 12418-12426. https://doi.org/10.1002/2017GL07567

678 Vigaud, N., A. Robertson, and M. Tippett, 2018: Predictability of recurrent weather regimes over 679 North America during winter from submonthly reforecasts. Mon. Wea. Rev., 146, 2559-2577, 680 doi:10.1175/MWR-D-18-0058.1.

681 Wang, L., A. Robertson, 2018: Week 3-4 Predictatability over the United States assessed from 682 two operational ensemble prediction systems. Climate Dynamics.

683 https://doi.org/10.1007/s00382-018-4484-9

684 Warner, T.T., 2011: Numerical Weather and Climate Prediction. Cambridge University Press, 685 550pp.

686 Wick, G., P. Neiman, F. Ralph, and T. Hamill, 2013: Evaluation of forecasts of the water vapor 687 signature of atmospheric rivers in operational numerical weather prediction models. Wea. 688 Forecasting, 28, 1337-1352, doi:10.1175/WAF-D-13-00025.1.

689 Wilks, D. S., 2011: Statistical Methods in the Atmospheric Sciences. 3rd ed. Elsevier, 676 pp.

690 Wilson, L. J. and Vallee, M. (2002). The Canadian updateable model output statistics (UMOS) 691 system: Design and development' tests. Weather and Forecasting, 17, 206-222.

692 Wilson, L. J. and Vallee, M. (2003). The Canadian updateable model output statistics (UMOS) 693 system: Validation against' perfect prog. Weather and Forecasting, 18, 288-302. 
694 Zhong, Q., J. Li, L. Zhang, R. Ding, and B. Li, 2018: Predictability of tropical cyclone intensity 695 over the Western North Pacific using the IBTrACS dataset. Mon. Wea. Rev., 146, 2741-2755, 696 doi:10.1175/MWR-D-17-0301.1.

697

698

699

700

701

702

703

704

705

706 


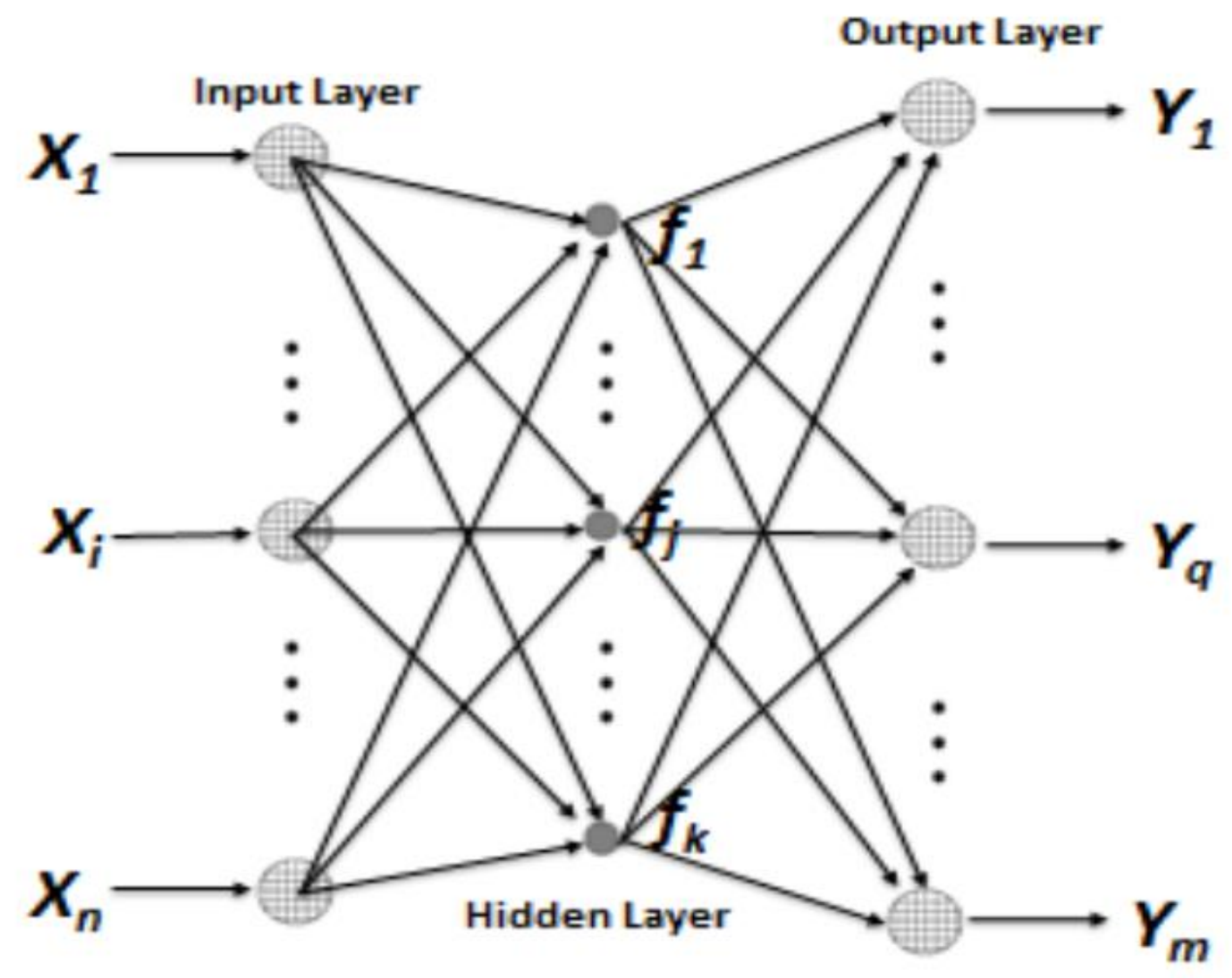

708 Fig.1 The simplest NN with one hidden layer and linear neurons in the output layer. The hidden

709 layer derives nonlinear transformations of the inputs and then linear combinations of these

710 nonlinear transformations are used to model the outputs. 


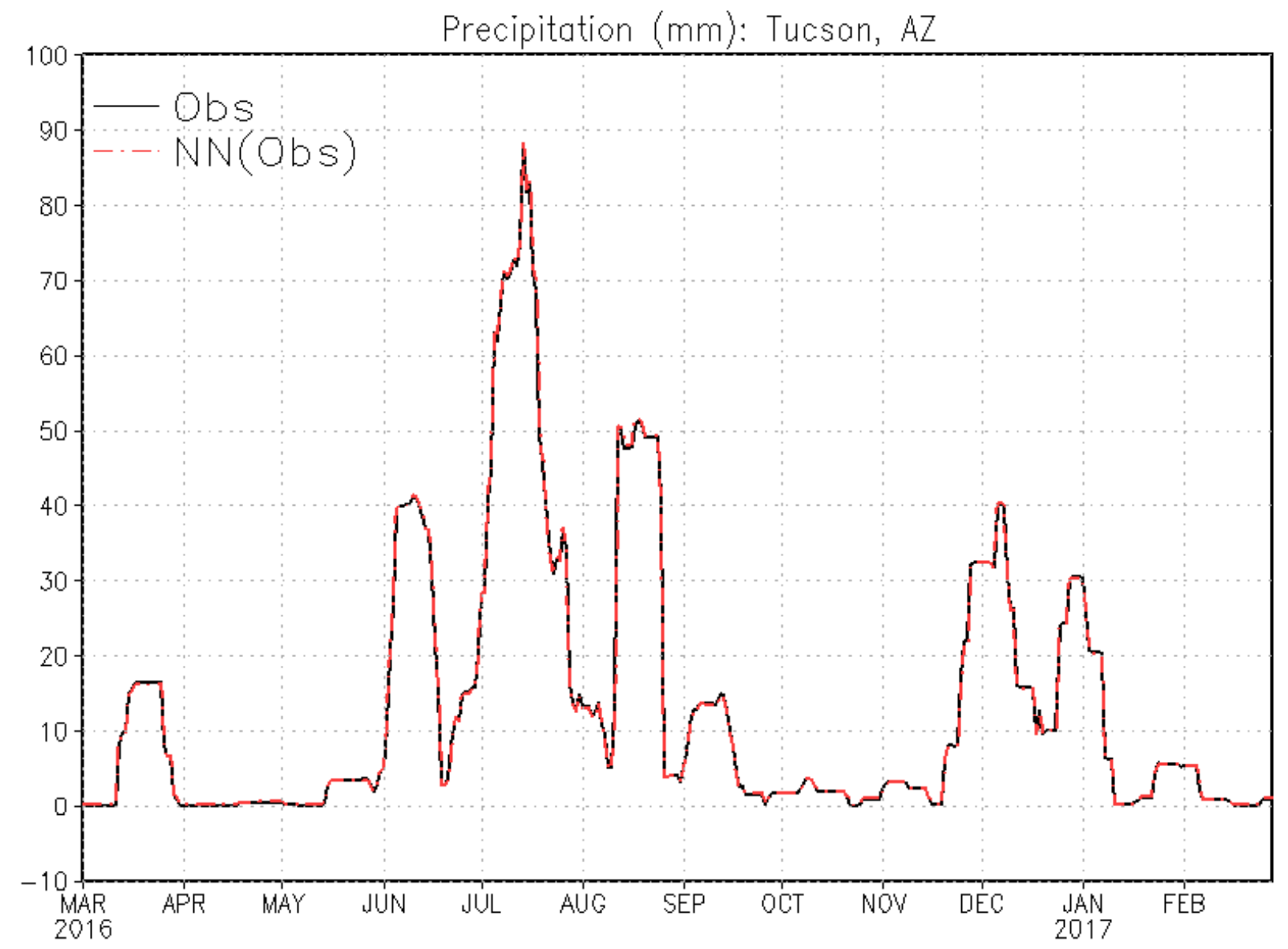

713 Fig.2 Time series of the observed 2 week total precipitation from Tucson, AZ (black line) and

714 the independent forecast Week 3 4 total precipitation (red line) (with observed precipitation

715 input) from NN-1 at same location and same period.

716

717

718 


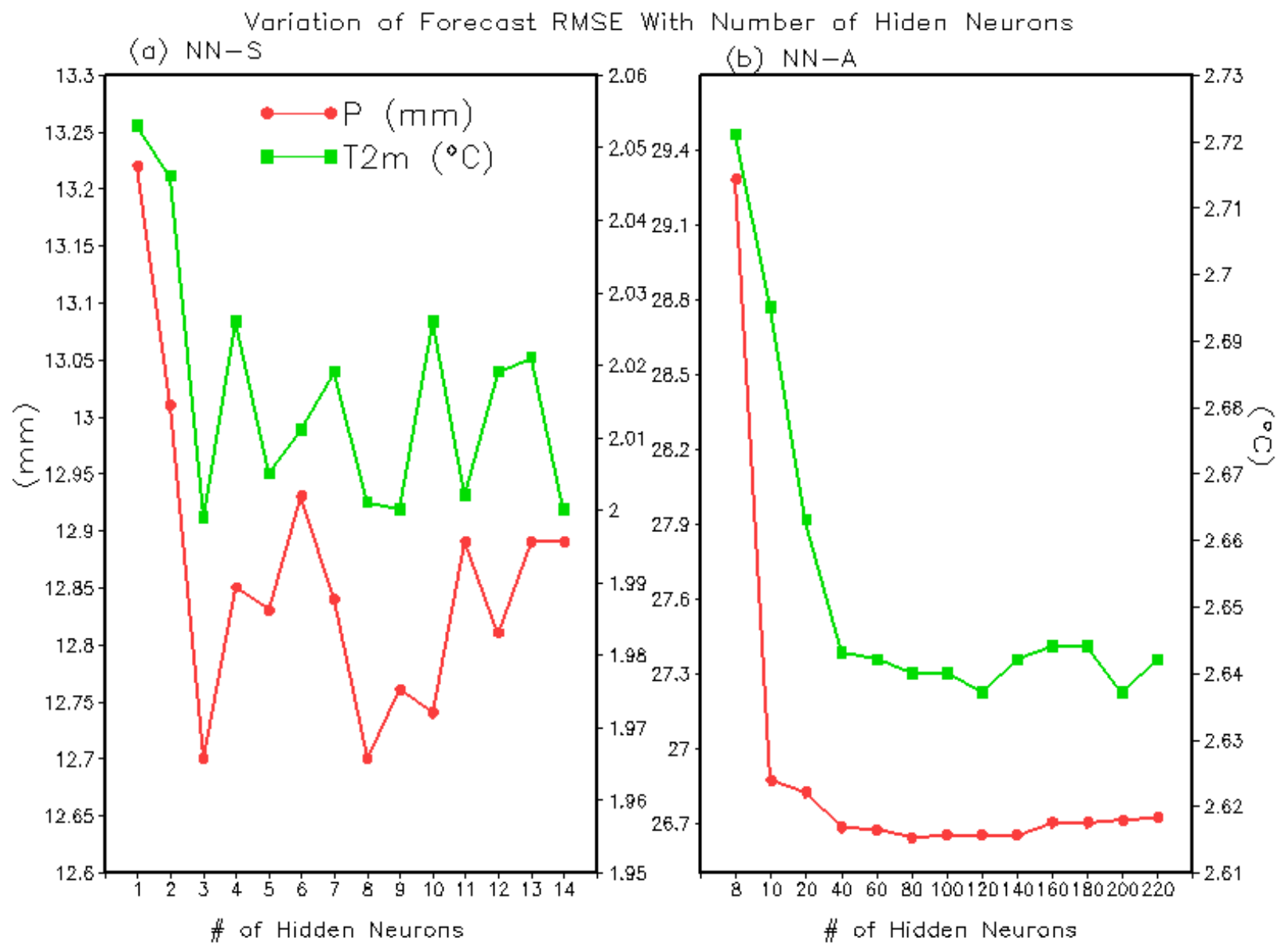

Fig.3 The mean Week 3 4 forecast RMSE (left scale in $\mathrm{mm}$ for $\mathrm{P}$ and right scale in ${ }^{\circ} \mathrm{C}$ for $\mathrm{T} 2 \mathrm{~m}$ )

721 as a function of hidden neurons $(k)$ on the independent forecasts from (a) the NN-S on randomly

722 selected 9 neighbor points and (b) the NN-A on the CONUS domain. Training period: Jan.1,

7231999 to Dec.2016. Validation period: Jan.1 2017 to Dec.31, 2018). 
Time Series of Week $3 \sim 4$ Prop $(\mathrm{mm})$
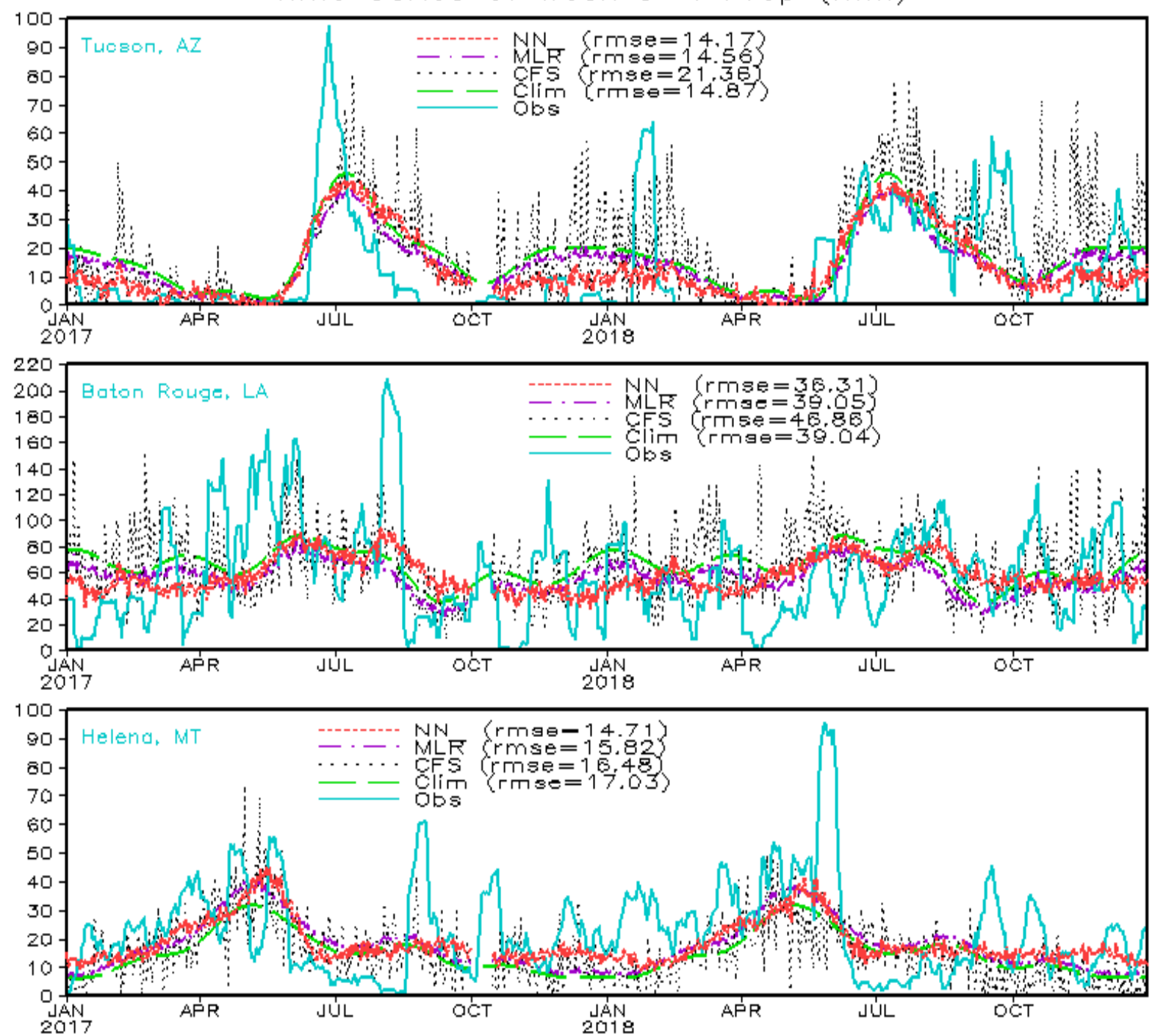

725 Fig.4 Time series of daily Week 3 4 total P on independent data by: NN-A forecasts (red dash),

726 MLR forecasts (purple dot-dash), bias corrected CFS forecasts (black dot), observed climatology

727 (green long dash) and observation (light blue solid) at 3 randomly selected locations. The values of RMSE are the averages over the two years. 

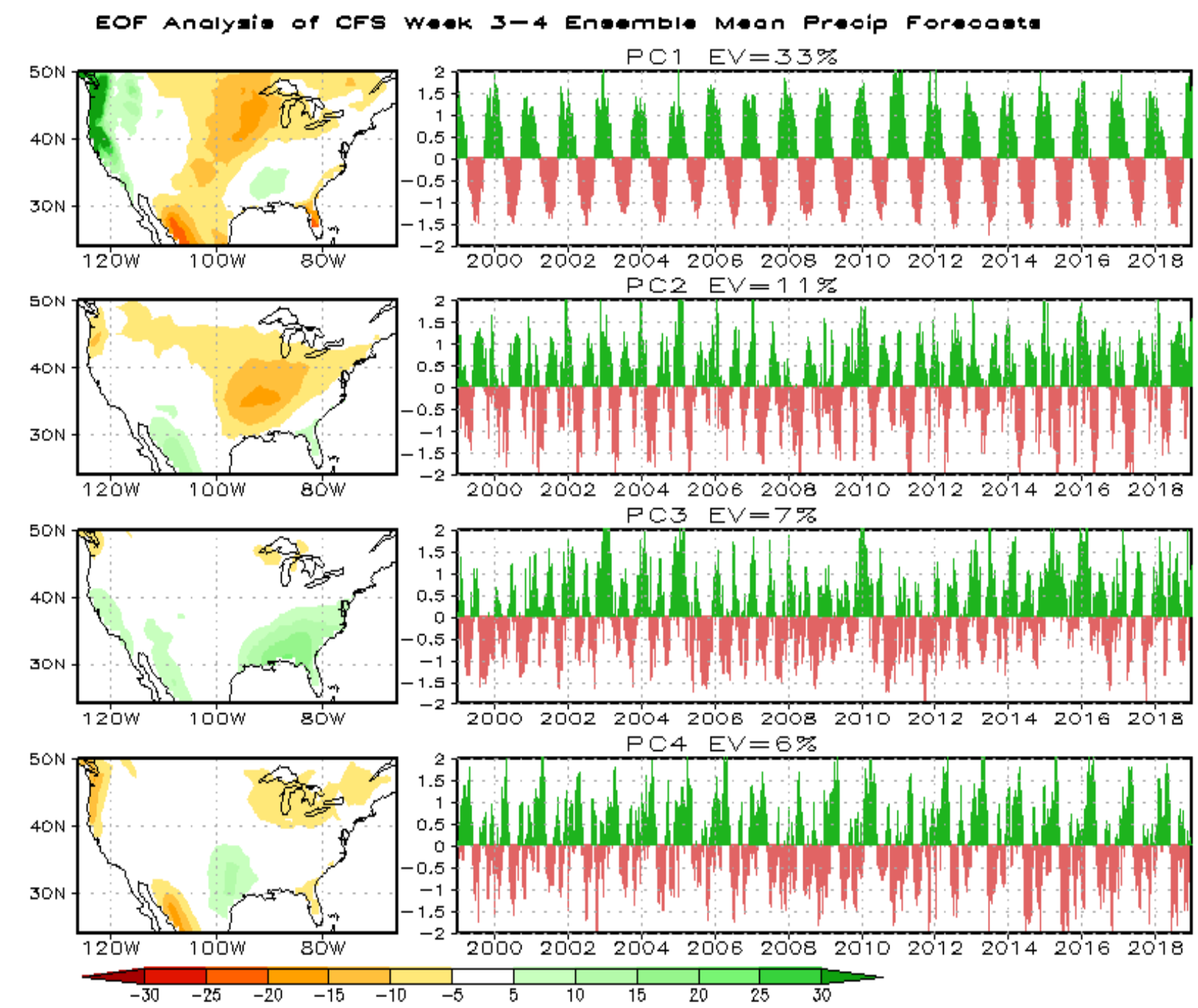

Fig.5 The first 4 leading EOF patterns (scaled by the RMS value of the associated PCs and units

731 are in millimeters) and their correspondent time series (normalized to unit variance) from bias

732 corrected CFS ensemble mean (average of 00Z, 06Z, 12Z and 18Z) Week 3 4 forecast total 733 precipitation. 


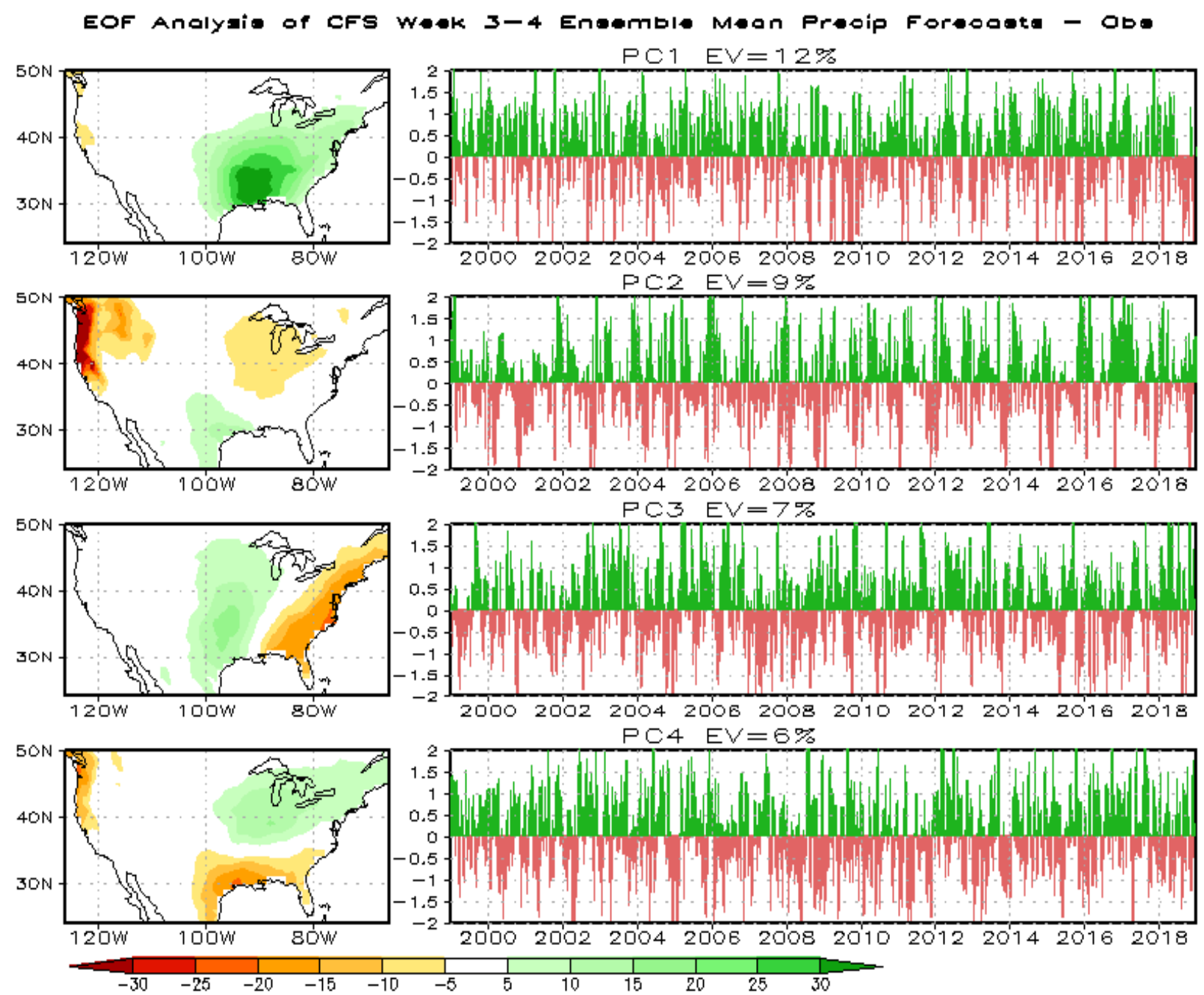

Fig.6 The first 4 leading EOF patterns (scaled by the RMS value of the associated PCs and units

736 are in millimeters) and their related time series (normalized to unit variance) from forecast errors

737 (bias corrected CFS Week 3 4 ensemble total P minus observation) 

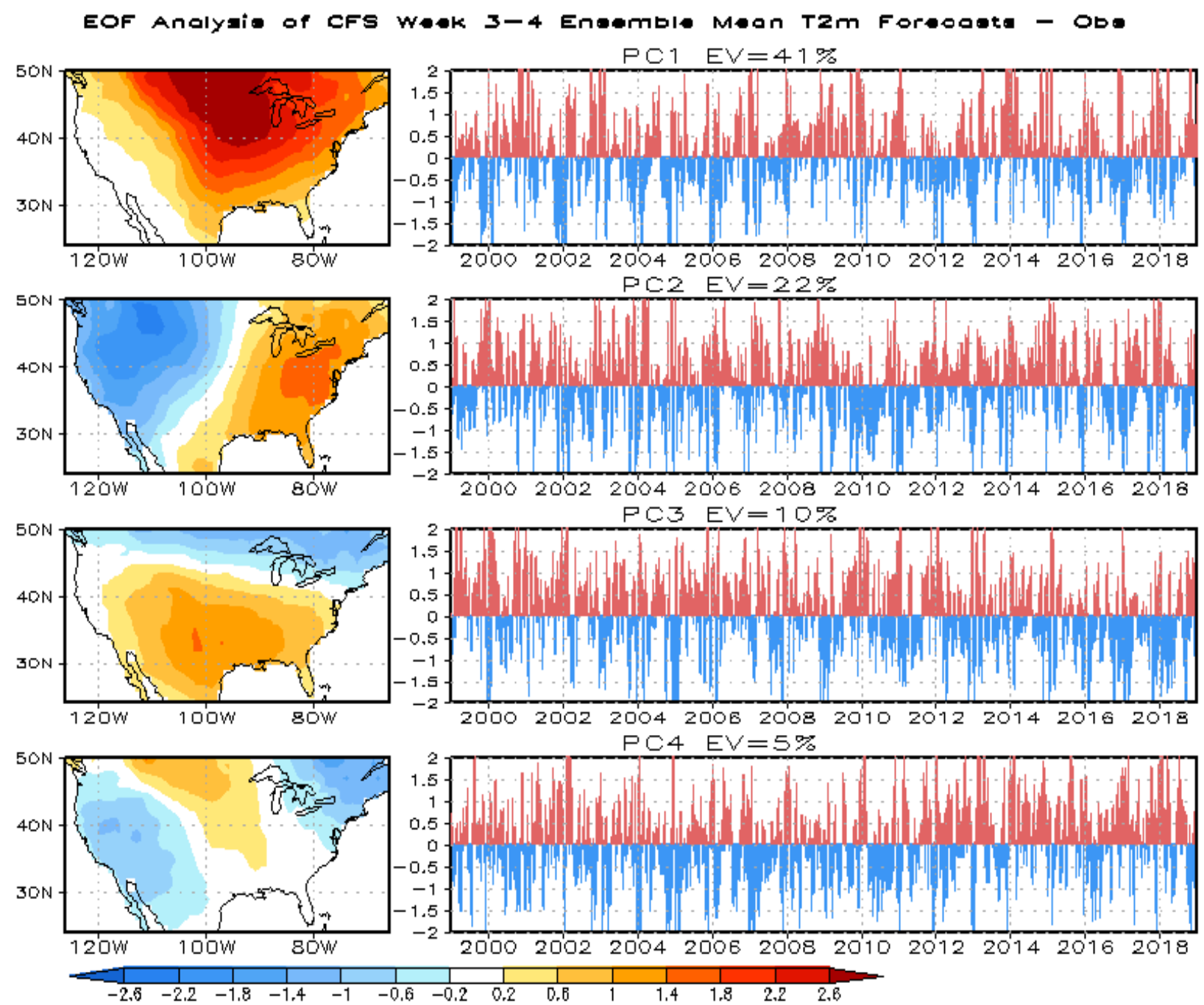

739 Fig.7 The first 4 leading EOF patterns (scaled by the RMS value of the associated PCs and units

740 are in degree C) and their related time series (normalized to unit variance) from forecast errors

741 (bias corrected CFS Week 3 4 ensemble mean T2m minus observation) 
Forecast WK $3 \sim 4$ Prcp RMSE $(\mathrm{mm})$ \& AC
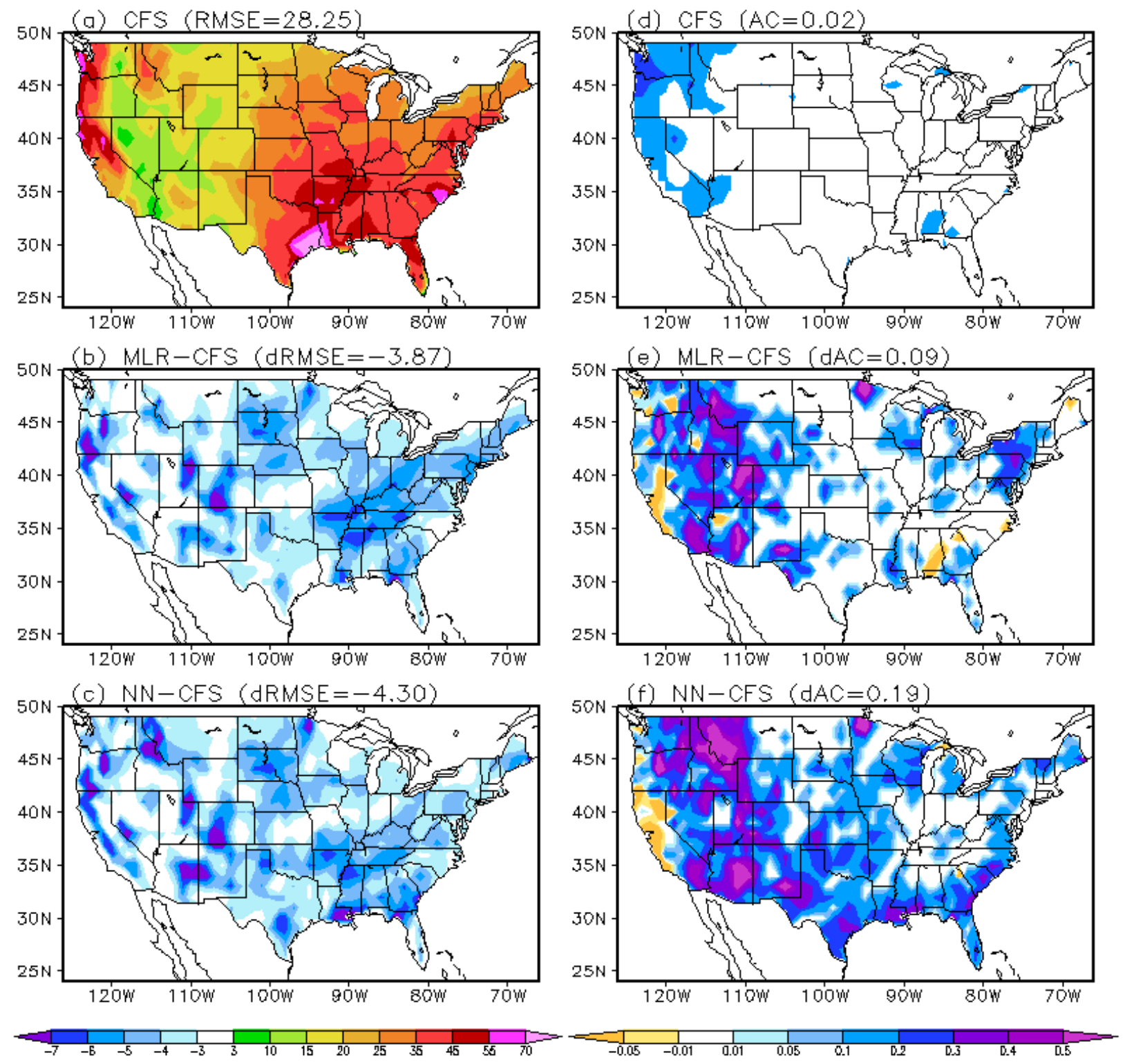

743 Fig.8 The RMSE (left panel) and AC (right panel) of daily Week 3 4 P by (a,d) Bias corrected

744 CFS forecasts, (b,e) RMSE differences (dRMSE) and AC differences (dAC) between CFS and

745 MLR forecasts, (c,f) same as (b,e) but between CFS and NN forecasts. Training period: Jan. 01,

7461999 to Dec. 31, 2016. Testing period: Jan.1 2017 to Dec. 31, 2018. The values in subtitle are

747 the averages over the CONUS domain. For the $\mathrm{AC}$ and $\mathrm{AAC}$, the shaded regions exceed $99 \%$

748 confidence level. 
Forecast WK $3 \sim 4$ T2m RMSE $\left({ }^{\circ} \mathrm{C}\right)$ \& $A C$
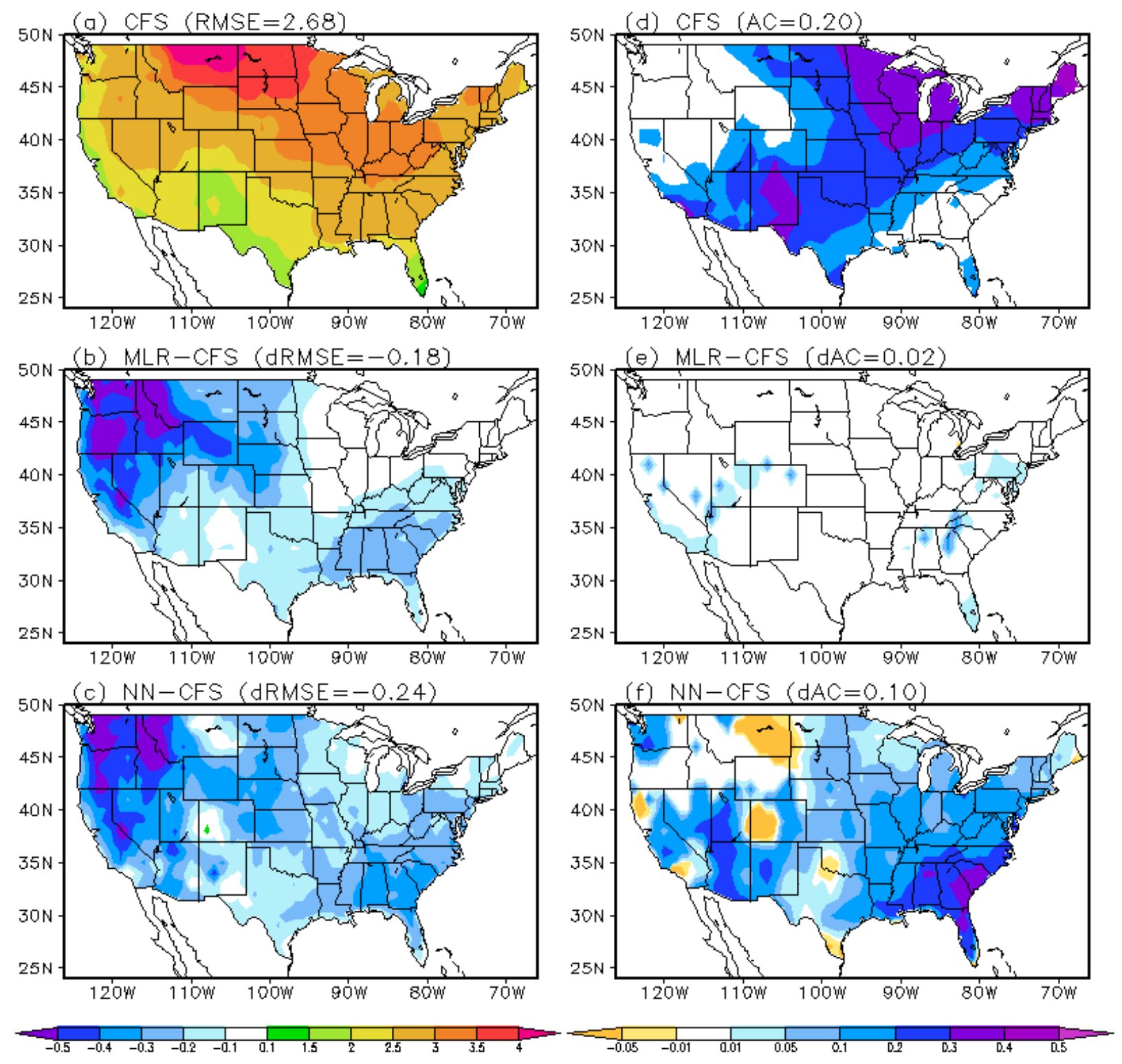

750 Fig.9 The RMSE (left panel) and AC (right panel) of daily Week 3 4 T2m by (a,d) Bias

751 corrected CFS forecasts, (b,e) RMSE differences (dRMSE) and AC differences (dAC) between

752 CFS and MLR forecasts, $(\mathrm{c}, \mathrm{f})$ same as $(\mathrm{b}, \mathrm{e})$ but between CFS and NN forecasts. Training period:

753 Jan. 01, 1999 to Dec. 31, 2016. Testing period: Jan.1 2017 to Dec. 31, 2018. The values in

754 subtitle are the averages over the CONUS domain. For the $\mathrm{AC}$ and $\mathrm{dAC}$, the shaded regions

755 exceed $99 \%$ confidence level. 

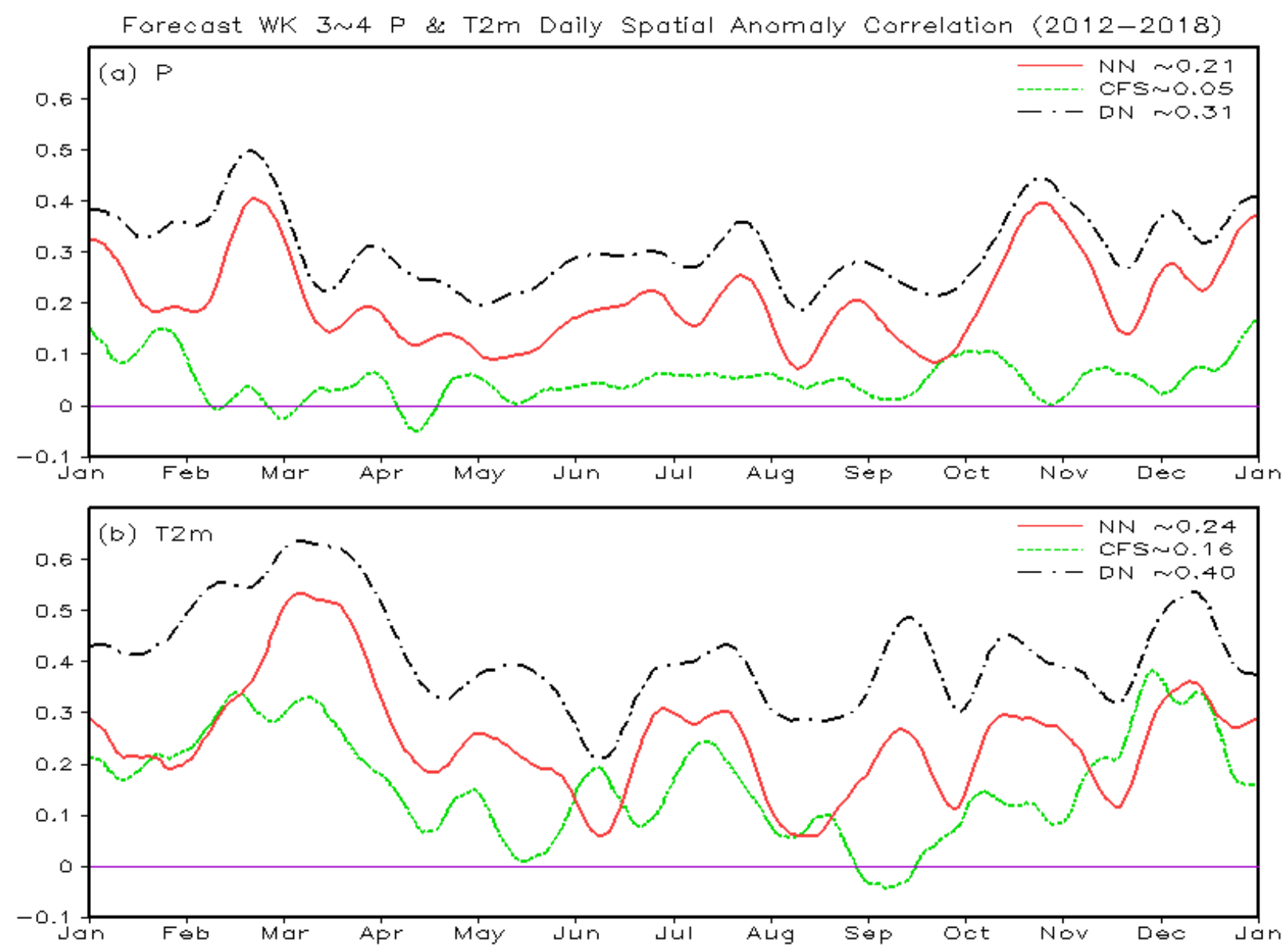

757 Fig.10. Mean time series of the daily Week 3-4 P and T2m spatial anomaly correlations over the

758 CONUS (5 day running mean applied) among (1) NN (red): independent NN forecasts (Test 3)

759 and observations, (2) CFS (green): bias corrected CFS forecasts and observations, and (3) DN 760 (black): dependent NN (all data from Jan. 1999 to Dec. 31, 2018 used for NN training) and 761 observations. Validation period: from Jan. 01, 2012 to Dec.31, 2018. 
Observed and Forecast WK 3w4 Prcp Anomalies (mm) Feb.11, 2017
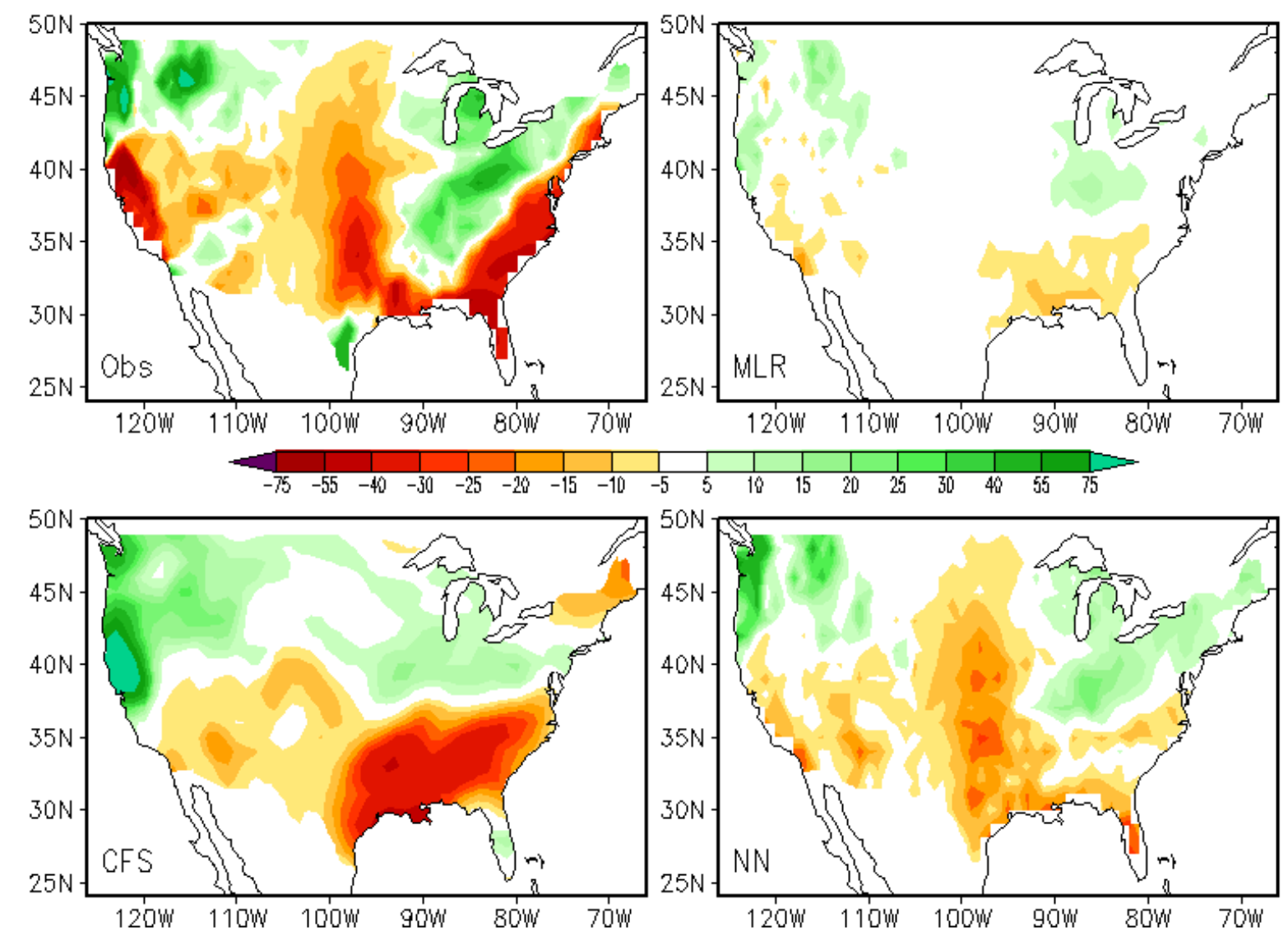

762

763 Fig. 11 The observed (Obs), CFS, MLR and NN forecast Week 3-4 P anomalies on Feb. 11, 7642017. 
Observed and Forecast WK 3 4 Prcp Anomalies (mm) Jul.25, 2017
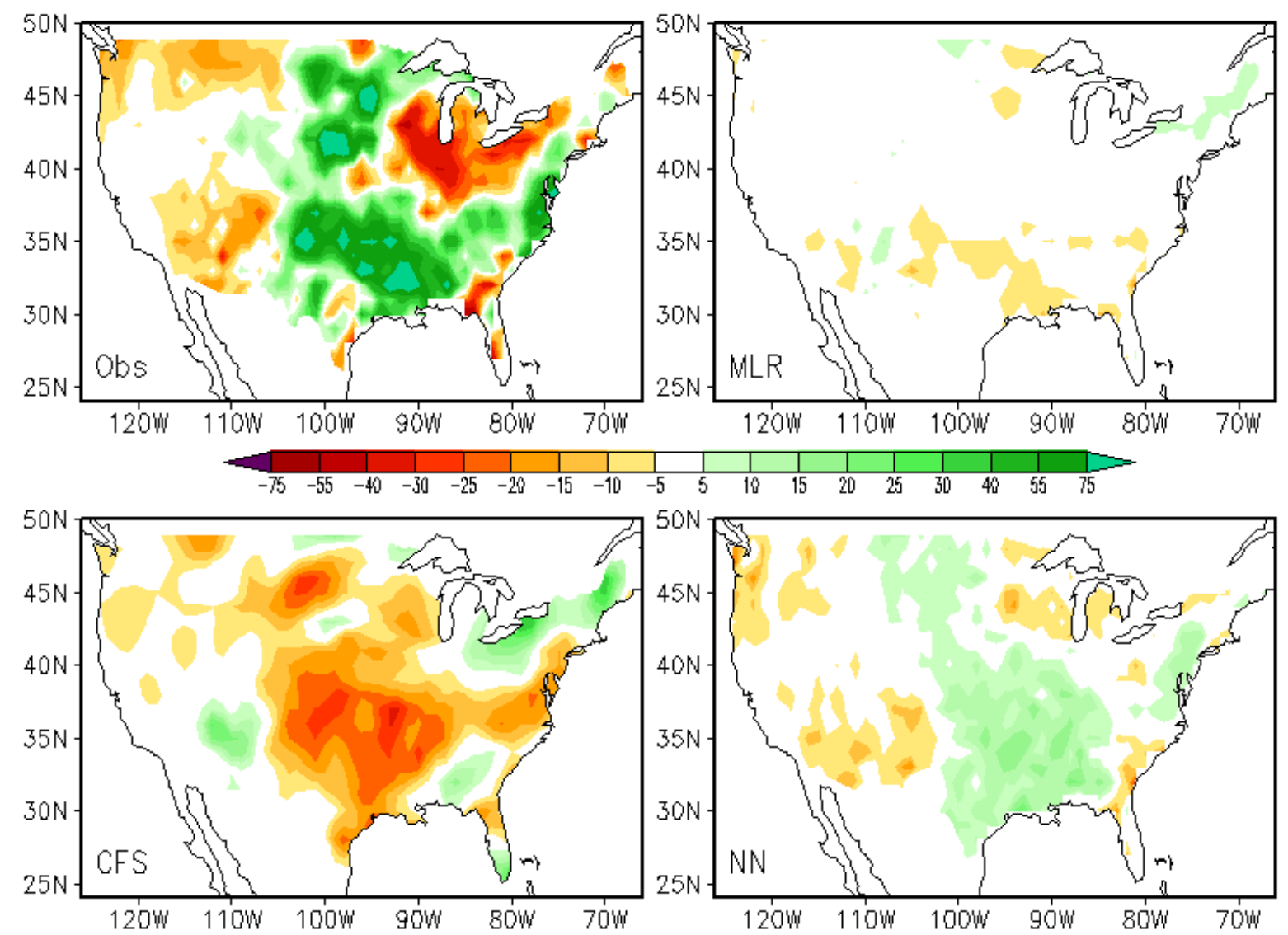

765

766

767 Fig. 12 Same as Fig. 11 but for Jul. 25, 2017. 
Observed and Forecast WK 3 4 T2m Anomalies ( ${ }^{\circ} \mathrm{C}$ ) on Mar.15, 2018
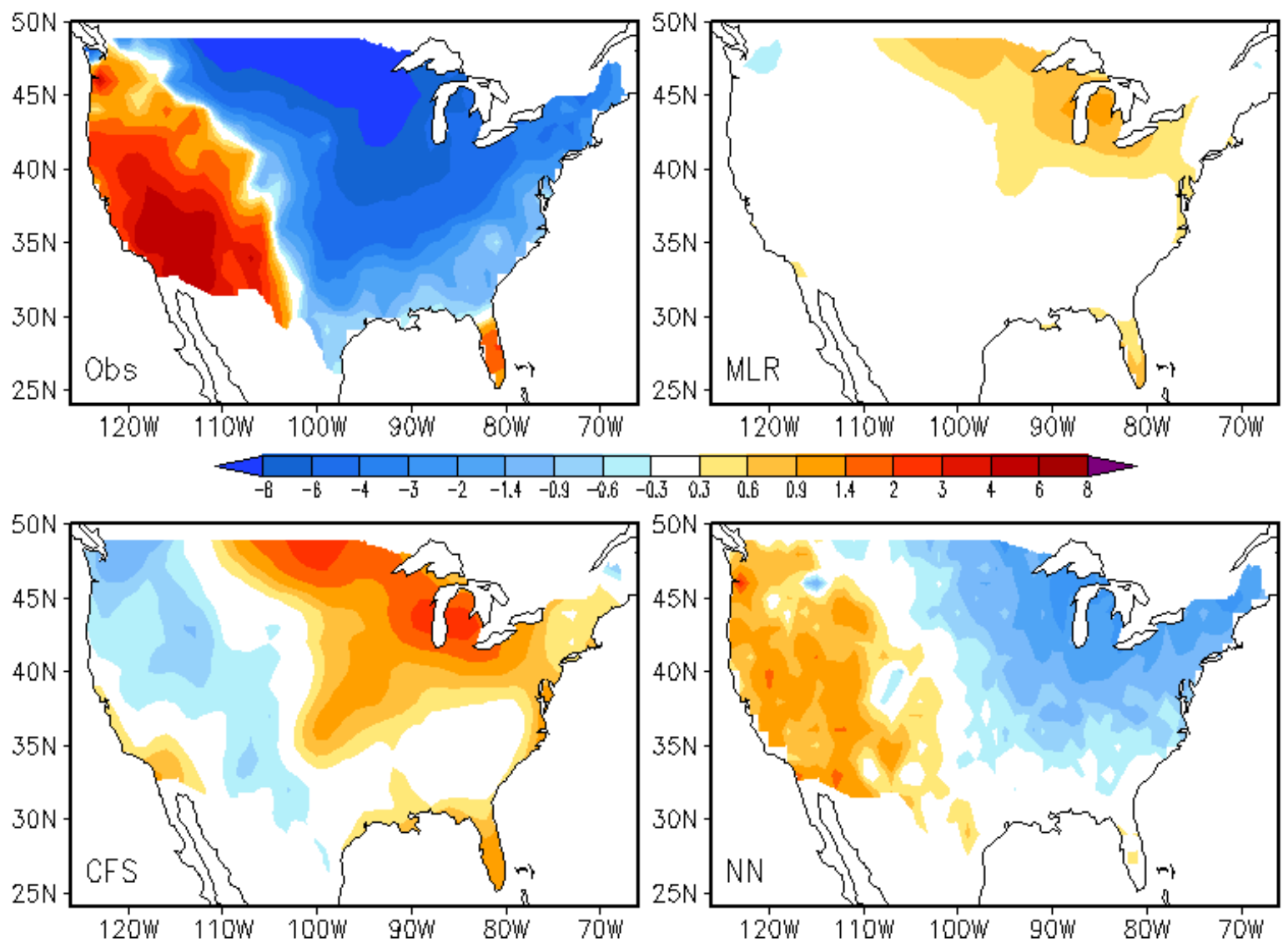

Fig. 13 The observed (Obs), CFS, MLR and NN forecast Week 3-4 T2m anomalies on Mar. 15, $770 \quad 2018$. 
Observed and Forecast WK 3 4 T2m Anomalies ( ${ }^{\circ} \mathrm{C}$ ) on Aug.10, 2018
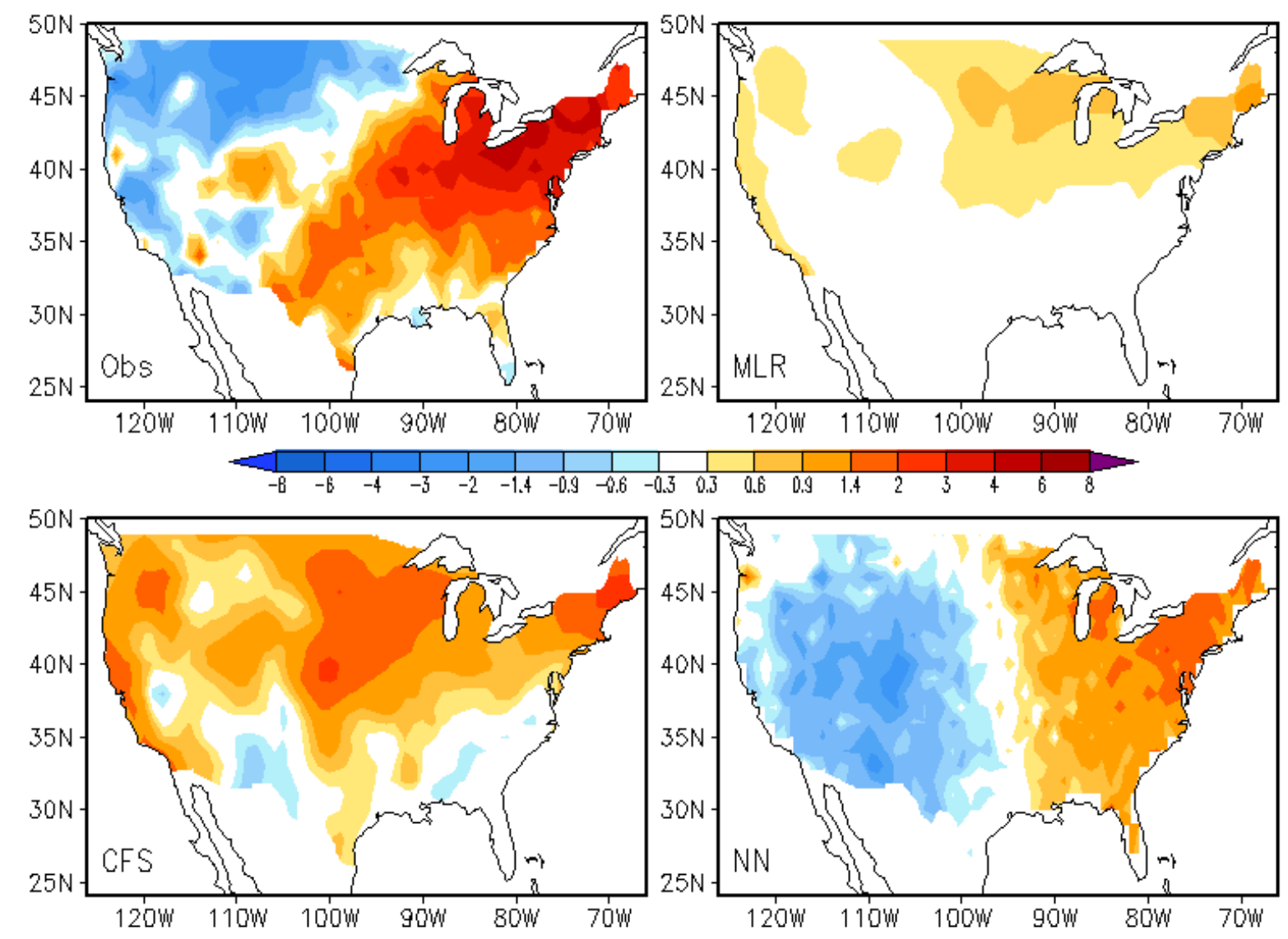

771

772 Fig. 14 Same as Fig. 13 but for Aug. 10, 2018. 